\title{
6 Einführung: Zur Analyse von Positionierungen im Kontext von Serienkommunikation
}

In Abschnitt 3.3 wurden folgende Merkmale - (1) Kontextgebundenheit, (2) Interaktivität und Relationalität, (3) Dynamik, (4) Gegenstandsbezogenheit, (5) (potentielle) Ubiquität, (6) Explizitheit und Intensität sowie (7) Räumlichkeit als grundlegend für Positionierungen dargelegt. Auf der Basis dieser Beschreibung wird im Folgenden in das für diese Studie entwickelte Analysekonzept von Positionierungen im Kontext von Serienkommunikation eingeführt. Dafür wird zunächst dargestellt, wie die Teilnehmenden die Gesprächssituation kontextuell behandeln (Abschnitt 6.1), welche interaktiven Ziele (Abschnitt 6.2) und welche kommunikativen Aufgaben (Abschnitt 6.3) sich rekonstruieren lassen. Es wird gezeigt, wie im Kontext der Daten Bewertungen und Wissenskommunikation miteinander zusammenhängen (Abschnitt 6.4). Anschließend wird der Fokus auf den Gesprächsgegenstand gelenkt (Abschnitt 6.5) und es werden zunächst Ressourcen (Abschnitt 6.5.2) beschrieben, mit denen die Jugendlichen sich in Bezug auf Serien(rezeption) positionieren, bevor dargestellt wird, wie die Gespräche um den Gegenstand herum organisiert und strukturiert werden (Abschnitt 6.5.3), wobei der Gegenstand hier als Positionierungsobjekt (Abschnitt 6.5.1) bezeichnet wird. Diese Ausführungen bilden die Grundlage für den analytischen Zugriff auf die vorliegenden Daten (Abschnitt 6.6), der in Kapitel 9 in Form einer Fallstudie zusammengeführt wird.

\subsection{Kontextualisierung der Gesprächssituation}

In diesem Abschnitt werden Kontextualisierungshinweise herausgearbeitet, die Aufschluss darüber geben, wie die Situation von den Teilnehmenden selbst hergestellt, definiert und interaktiv bearbeitet wird (vgl. u.a. Spranz-Fogasy und Deppermann 2004: 1153, Deppermann 2008: 14, Gülich und Mondada 2008: 17, Hausendorf 2007: 407). Dafür wird auch analysiert, wie die Teilnehmenden das participation framework (vgl. zur Erklärung des Begriffs Abschnitt 4.1) der Interaktion herstellen und modifizieren, wie sie das Gespräch organisieren (Abschnitt 6.1.1) und welche Rahmenbedingungen in welcher Form kontextualisiert werden (Abschnitt 6.1.2).

\subsubsection{Die Gruppendiskussion als aufgabenorientierte Interaktion}

Die Interaktionssituation als arrangierte Gesprächsform (siehe dazu auch Abschnitt 5.3) findet insofern Berücksichtigung, als über die Analyse der Kontex- 
tualisierungshinweise rekonstruiert wurde, dass es sich bei den vorliegenden Gesprächsdaten um aufgabenorientierte Kommunikation ${ }^{1}$ handelt. Die Aufgabe wird von der Forscherin eingeführt, indem sie erläutert, dass sie an den serienbezogenen Präferenzen der Jugendlichen interessiert ist (zur detaillierteren Beschreibung der Erhebungssituation vgl. Ausführungen in Abschnitt 5.1.1). Im Folgenden wird zunächst analysiert, wie die Teilnehmenden den Gesprächsbeginn gestalten und in eine fokussierte Interaktion (Goffman 1963: 24) übergehen, da über die Analyse von Initialsequenzen oft identifiziert werden kann, wie Interagierende geteiltes Verständnis einer Situation herstellen (vgl. Ausführungen in Abschnitt 5.2).

Der folgende Ausschnitt stammt aus dem Gespräch zwischen sechs Schüler^innen des 11. Jahrgangs einer Gesamtschule. Vergleichbare Einstiege finden sich aber auch in den meisten anderen Daten. Für einen Überblick über das GesamtKorpus kann die Tabelle 5.1 hinzugezogen werden. Die Transkripte sind BasisTranskripte nach GAT 2 (Selting u. a. 2009); die Konventionen können im Anhang nachgelesen werden. Die Transkriptsegmente sind fortlaufend durchnummeriert, so dass im Verlauf der Arbeit auf sie verwiesen werden kann. Anschließend folgt eine Überschrift, die aus einem für den Analysefokus relevanten Zitat des Ausschnitts besteht. Rechts unter dem Ausschnitt ist der Verweis auf das Kürzel des Gesprächs sowie die Minutenangabe der Sequenz. Im Anhang können alle Gespräche eingesehen werden und über das Kürzel sowie die entsprechenden Zeilennummern eindeutig identifiziert werden. Wörtliche Transkriptzitate im Fließtext werden durch doppelte Anführungszeichen und Kursivschreibungen sowie die Angabe der Zeilennummer(n) gekennzeichnet.

(1) dann könnt ihr LOSlegen und ich lass euch jetzt alleine

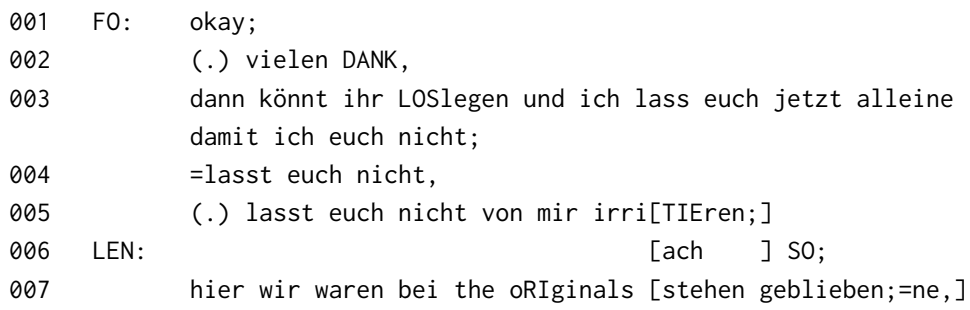

1 Denn auf struktureller Ebene finden sich Parallelen zu beispielsweise der von Filipi (2016) identifizierten Struktur, die Schüler*innen bei der Bearbeitung einer map task etablieren, sowie zu Ergebnissen von Schaller (2012). 
MAR :

[wollen wir

] ERSTmal,

009

010 LEN :

was GUCKT ihr denn so für [serien; ]

[ja ] was guckt IHR eigentlich so;

MNG11, 0:53-1:21

Mittels der Diskursmarker „okay;“ (Z. 001), „vielen DANK,“ (Z. 002), die beide als „sequence-closing thirds“ (Schegloff 2007: 118) fungieren, und der Aufforderung „dann könnt ihr LOSlegen“ (Z. 003) leitet die Forscherin den Beginn des Gesprächs ein. Zusätzlich kündigt sie an, den Raum zu verlassen (Z. 003-005). In einer kurzen Überlappungssequenz quittiert Leni diese Ankündigung mit dem change-of-state token „ach SO;“ (Z. 006). Wie Golato und Betz (2008: 10) zeigen, fungiert ein „ach so" an dritter Position innerhalb einer Reparatursequenz als Signal für den Abbau epistemischer Asymmetrie (siehe auch Abschnitt 4.3). Die Reaktion auf die Ankündigung des Raumverlassens weist also darauf hin, dass das Verhalten der Forscherin als eher unerwartet eingestuft wird. An dieser Stelle wird die Abwesenheit der Forscherin jedoch als nicht weiter problematisch behandelt, da Leni direkt mit ihrer Äußerung fortfährt und thematisch in das Gespräch einsteigt, während das Verhalten der Forscherin in anderen Fällen expliziter thematisiert (vgl. z.B. Anhang II: EMS53, Z. 022-024) bzw. zum Teil aber auch positiv beurteilt (Anhang I: EMS51, Z. 017-019) wird. Schließlich signalisieren sich die Teilnehmenden wechselseitig verbal und nonverbal ihre Gesprächsbereitschaft (Z. 007-010) und etablieren mit der Serie THE ORIGINALS ein erstes gemeinsames Thema (Z. 007), das der Instruktion seitens der Forscherin entspricht. Dabei verweist Leni auf einen ihr und Josefine bekannten Kontext außerhalb der aktuellen Gesprächssituation: Beide haben sich zuvor offenbar bereits über die Serie ausgetauscht. So können die Gespräche auch Spuren vorheriger Interaktionen aufweisen.

Lenis Thema wird von Martha jedoch im Rahmen einer Überlappungssequenz abgelehnt (Z. 008) und als offenere Frage, die an einen größeren Personenkreis adressiert ist, umformuliert (Z. 009). Daran zeigt sich eine Orientierung an der Aufgabenstellung der Forscherin, sich als gesamte Gruppe über rezipierte Serien auszutauschen (vgl. auch Abschnitt 6.1.2). Die Instruktion, über Serien zu sprechen, impliziert aber auch, dass möglichst keine anderen Themen zum Gesprächsgegenstand gemacht werden. Das folgende Beispiel zeigt, wie dieses Verständnis von den Gruppenmitgliedern expliziert und ausgehandelt wird: 


\section{(2) wir DÜRfen nicht über filme reden;}

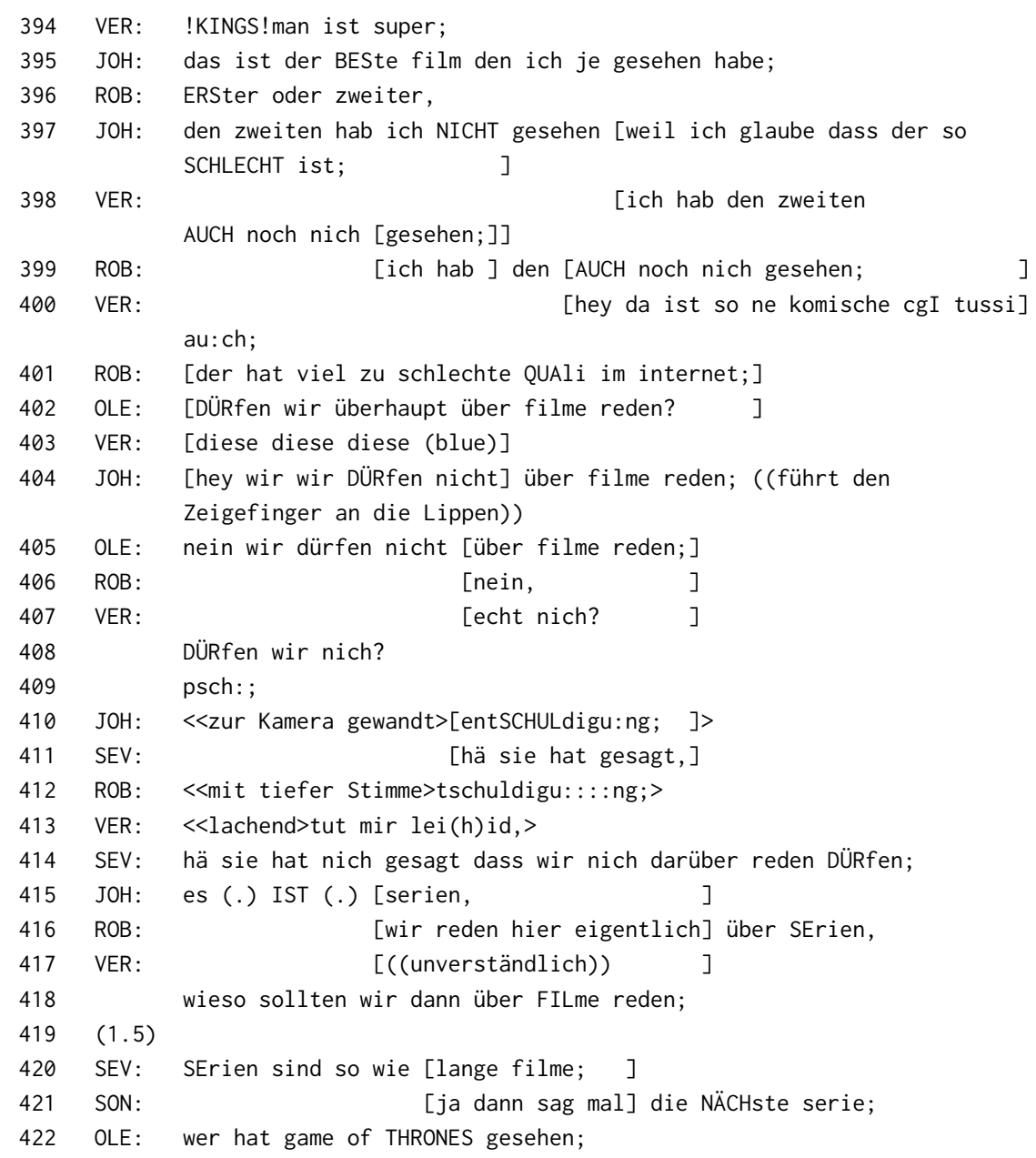

Der Auszug stammt aus dem Gespräch, nachdem bereits ca. 25 Minuten vergangen sind. Zuvor hatten die Jugendlichen über verschiedene Medienprodukte gesprochen, wobei Johann den Film KINGSMAN nennt. Es entwickelt sich eine Sequenz, in der der Film und dessen Fortsetzung miteinander verglichen und unterschiedlich bewertet werden (Z. 396-401). Schließlich stellt Ole - überlappend mit einigen anderen Interaktionsbeteiligten - die Frage, ob sie „überhaupt über filme reden [DÜRfen]?“(Z. 402). Während Verena noch mit der Bewertungsinteraktion fortfährt, 
greifen Johann und Robert Oles Aussage auf und ratifizieren sie (Z. 404-406); nonverbal unterstützt mit einer „Leise-Geste“ (Z. 404), in die Verena nach einer kurzen Bestätigungssequenz einsteigt (Z. 409). In einem humorvollen Interaktionsmodus wird nun der vermeintliche ,Fehltritt‘ der Gruppe performativ inszeniert, indem Johann sich vorbeugt und ein langgezogenes „entSCHULdigu:ng“ in die Kamera spricht (Z.410), was die anderen amüsiert kommentieren und imitieren (Z. 412-413). Sevcan stellt die Legitimation dieser Entschuldigungssequenz in Frage, indem sie konstatiert: „hä sie hat nich gesagt dass wir nich darüber reden DÜRfen“ (Z. 414). Mit dem Personalpronomen „sie“ verweist sie auf die (abwesende) Forscherin, womit sie ihr implizit die Rolle derjenigen zuweist, die die Aufgabe stellt und die thematischen Grenzen des Gesprächs festlegt. Johann blickt daraufhin auf den eingangs ausgefüllten Fragebogen (vgl. Abschnitt 5.1.1), der neben ihm auf dem Tisch liegt, und bekräftigt mit stakkatoartiger Intonation, dass es in der Studie um Serien gehen soll (Z. 415). Robert und Verena stellen implizit Serien und Filme als kontrastive Kategorien einander gegenüber, die für Sevcan offenbar weniger dichotom sind (Z. 416-419, vgl. dazu auch die Analyse in Abschnitt 8.1.1). Die Sequenz wird beendet, indem Sonja Robert mittels deiktischer Geste auffordert, die nächste Serie zum Gesprächsthema zu machen (Z. 421). Allerdings folgt stattdessen Ole als nicht-adressierter Rezipient dieser Aufforderung und fragt, ob jemand die Serie GAME OF Thrones gesehen habe (Z. 422).

Letzteres verweist auf die prototypische Strukturierung der Gespräche, die entweder um eine konkrete Serie oder abstraktere, aber serienbezogene Aspekte herum organisiert sind (vgl. dazu ausführlicher Abschnitt 6.5.3). Dennoch loten die Teilnehmenden bisweilen explizit die thematischen und inhaltlichen Grenzen des Gesprächs aus, woraus sich wiederum Rückschlüsse auf das Situationsverständnis ergeben. So wird die Forscherin als diejenige, die die Situation arrangiert und den thematischen Impuls gegeben hat, behandelt sowie als Instanz bei Fragen nach der ,Sagbarkeit‘ (vgl. auch Beispiel 4) hinzugezogen und ihr damit eine Lehrkräften ähnliche Rolle zugeschrieben. Da sie nicht die für Gruppendiskussionen erwartbare Moderationsrolle erfüllt, übernehmen die Teilnehmenden diese Funktion wechselseitig und temporär, um das Gespräch zu organisieren (z.B. MNG11, Z. 009010 (Beispiel 1; Anhang IX: OST11 Z. 001, Anhang VI: RHG10, Z. 410). Die Häufung von Frage-Antwort-Sequenzen legt nahe, dass die Teilnehmenden das Gespräch wie ein Gruppeninterview mit wechselseitigen diskursiven Rollen behandeln.

Für die Analyse der Positionierungen ergeben sich daraus folgende Konsequenzen: Die forscherinnenseitige Engführung auf den vorgegebenen Gesprächsgegenstand führt zu einer Vergleichbarkeit der Daten in dieser Hinsicht. Serien als Positionierungsobjekt (Abschnitt 6.5.1) bilden den zugrundeliegenden Referenzrahmen für die Interaktion, auf den die Teilnehmenden die meiste Zeit über Bezug nehmen und an dem sie sich gesprächsstrukturell orientieren. 


\subsubsection{Herstellen von Institutionalität, Öffentlichkeit und Kooperativität}

Im Folgenden werden weitere Kontextualisierungshinweise analysiert, die Aufschluss über die von den Beteiligten hervorgebrachten Rahmenbedingungen der Gespräche geben. So lassen sich Spuren der Institution Schule erkennen, die sowohl aufgrund der äußeren Umgebung naheliegen als auch punktuell von den Teilnehmenden selbst relevant gesetzt werden. Zugleich muss berücksichtigt werden, dass das Gespräch unter einem gewissen Grad von Öffentlichkeit stattfindet, was durch die Thematisierung und Adressierung der Kamera salient wird (vgl. auch Abschnitt 5.3). Eine wichtige Funktion nimmt das Prinzip der Kooperativität sowohl der Teilnehmenden untereinander als auch mit der (abwesenden) Forscherin ein. Anhand exemplarischer Fallbeispiele wird im Folgenden aufgezeigt, wie sich die Teilnehmenden an diesen drei Aspekten orientieren.

Die Aufnahmen finden in Räumlichkeiten der Schule statt, die Forscherin stellt sich als Mitarbeiterin der Universität vor (vgl. jeweils 5.1.1) und die Teilnehmenden behandeln das Gespräch als Resultat einer Instruktion (vgl. Abschnitt 6.1.1), die einen thematischen Rahmen vorgibt. Damit liegt es nahe, dass Institutionalität und ihre kommunikativen Besonderheiten (vgl. exemplarisch Ehlich 2009) implizit ,mitschwingen' und die Interaktion beeinflussen (können)². Das folgende Beispiel zeigt, wie Institutionalität interaktiv explizit sichtbar wird, als die Teilnehmerinnen zu Beginn des Gesprächs - nachdem die Forscherin den Raum verlassen hat - ihre Gesprächsorganisation diskutieren:

(3) wer ist denn dann die LEHrerin;

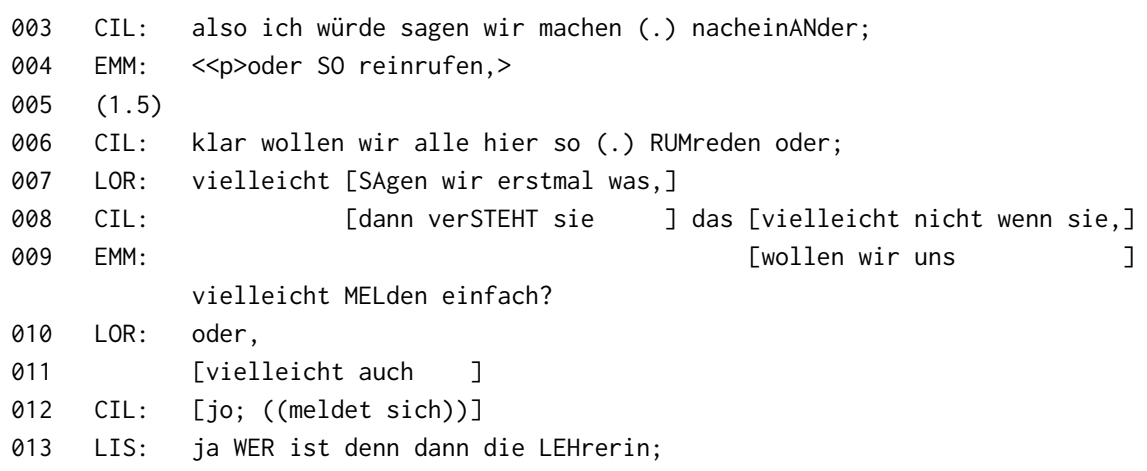

RGM07, 7:20-7:32

2 Wie Meer (2011: 29) argumentiert, ist eine grundlegende Trennung zwischen Alltag und Institution jedoch aus empirischer Sicht fraglich; vielmehr sollte jede „Kommunikation immer auf ihre institutionelle Einbindung und ihre Aufgaben- bzw. Zweckorientierung hin untersucht werden“. 
Cilia schlägt vor, nacheinander das Rederecht zu verteilen (Z. 003). Dem widerspricht Emmy, wenn auch sehr leise (Z. 004). Ihre Formulierung „reinrufen, “weckt dabei Assoziationen zur schulischen Organisation der Redebeiträge bzw. der gängigen Sanktionierung von Beiträgen, die nicht den Gesprächsregeln entsprechen. Cilia interpretiert Emmys Äußerung auch als Zurückweisung ihres Vorschlags mit einer zunächst als sarkastisch gerahmten Äußerung (Z. 006), die sie später sachlogisch mit dem Verweis auf die Auswertung der Daten seitens der Forscherin begründet (Z. 008). Daraufhin schlägt Emmy einen Kompromiss vor, der ebenfalls Spuren schulischer Interaktionsorganisation aufweist: „wollen wir uns vielleicht MELden einfach?“ (Z. 009). Die Gruppe greift Emmys Äußerung scherzhaft auf und spielt weiter auf institutionalisierte Kommunikation und deren mit spezifischen Rechten und Pflichten ausgestattete Rollen (,WER ist denn dann die LEHrerin;“) an.

Institutionalität scheint insbesondere Fragen der Gesprächsstrukturierung zu betreffen, denn tatsächlich gehen viele Gruppen - primär die jüngeren Schüler*innen (RGM07, SBH06 und RHG10) - meistens reihum vor und diskutieren nacheinander die Serien, die von den einzelnen Personen genannt werden. Für die Analyse von Positionierungen ist es deswegen sinnvoll, einzubeziehen, wem aktuell das Rederecht aufgrund der Gesprächsorganisation (nicht) zugewiesen wurde, um beispielsweise den Anspruch auf Erstbewertung und die epistemische Autorität (wie in den Analysen in Kapitel 7) dazu adäquat einschätzen zu können. Dennoch lässt sich der Institutionalitätsaspekt nicht durchgängig in den Daten rekonstruieren; zum Teil ähneln die Gespräche anderenorts eher lockerem PeerTalk, bei dem jede ${ }^{\star}$ j jederzeit das Rederecht beanspruchen kann und es zu vielen Überlappungen und Simultansprechen kommt (v.a. bei MNG11 und OST11).

Öffentlichkeit ist durch die Kamera sowie das Wissen um die nachträgliche Sichtung der Aufnahmen durch die Forscherin potentiell gegeben. Das zeigt sich unter anderem an Äußerungen, die die Kamera explizit thematisieren. In der folgenden Sequenz wird die nicht anwesende Forscherin über die Kamera adressiert:

(4) wenn du dich !HÖRT!;

$\begin{array}{lll}704 & \text { VER: } & \text { und äh::; } \\ 705 & & (-) \text { [das mit der DICken? } \\ 706 & & \text { die macht_äh nicht ALles?] } \\ 707 & \text { JOH: } & \text { [das ist voll die tolle SENdung; } \\ 708 & & \text { alle sind so Asozial; }] \\ 709 & \text { VER: } & \text { (.) }[\text { aber, } \\ 710 & \text { ROB: } & \text { [höhö Asozial; }] \\ 711 & \text { VER: } & \text { die [die macht ((unverständlich)) ] }\end{array}$




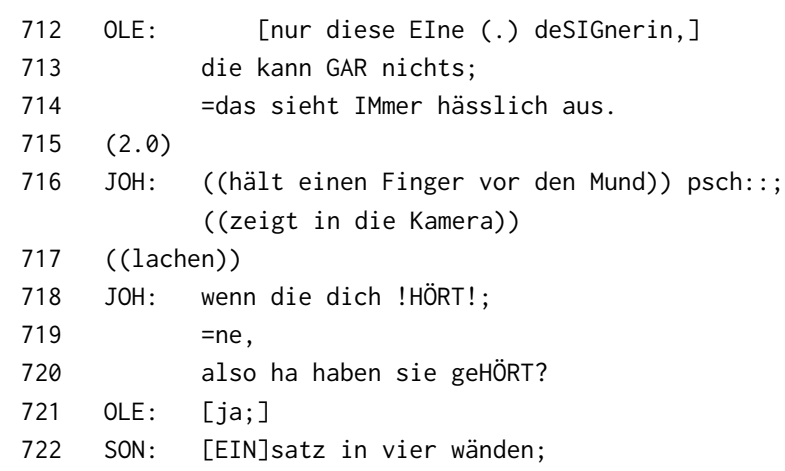

Dieser Ausschnitt ist kontextuell eingebettet in eine Sequenz, in der Verena nach dem Namen der Sendung EINSATZ IN VIER WÄNDEN sucht, deren Moderatorin sie als „die DICke“(Z. 705) bezeichnet, und Ole der Moderatorin zuschreibt, dass die „GAR nichts; “ könne und dass das „IMmer hässlich aus[sieht.]“ (Z. 713-714). Danach wird diese Bewertung im Rahmen einer Scherzinteraktion nachträglich als für die abwesende Forscherin durch die Kamera nachvollziehbar gerahmt und humorvoll sanktioniert. Zunächst wird der Forscherin die Rolle einer Lauscherin (eavesdropper im Goffman'schen (1981) Sinne) zugeschrieben, indem über sie gesprochen wird (Z. 718). Kurz darauf ändert sich das participation framework dahingehend, dass die Forscherin selbst über die Kamera ratifiziert und adressiert wird, da nicht über (,wenn die dich !HÖRT!; =ne, “, Z. 718-719) sie, sondern zu (,also ha haben sie geHÖRT?“, Z. 720) ihr gesprochen wird. Damit signalisieren die Jugendlichen, dass sie die Forscherin als spätere Beobachterin des Gesprächs antizipieren. Gleichsam setzen sie die Kamera als Dokumentations- und Speichermedium relevant, indem sie sich selbst für ,Normverstöße‘ maßregeln (vgl. für eine ähnliche Situation Anhang IX: OST11, Z. 237-265 oder auch Anhang VI: RHG10, Z. 712-727, als sich die Teilnehmenden mit ihren echten Namen statt den Alias-Namen ansprechen).

Das Prinzip der Kooperativität bedeutet auf der einen Seite, dass die Teilnehmenden versuchen, die Aufgabe möglichst gut für die Forscherin zu bearbeiten. Das zeigt sich zum Beispiel an Cilias Kommentar in Beispiel 3: „dann verSTEHT sie das vielleicht nicht“ (Z. 008). Aber auch andere gesprächsorganisierende Äußerungen weisen darauf hin: 
(5) dann ist das vielleicht mit der kamera n bisschen (.) EINFacher zu erkennen;

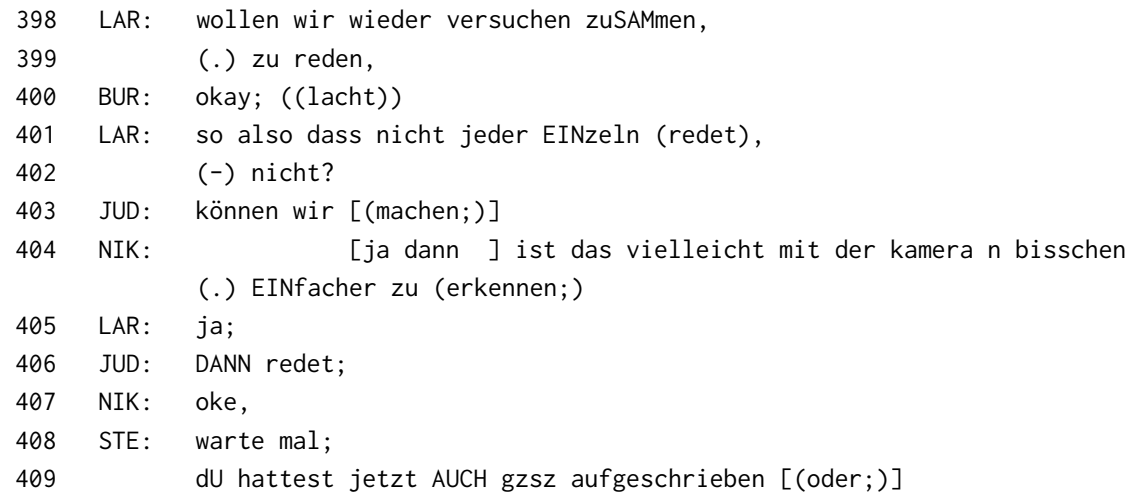

In dieser Sequenz versuchen Lara und Niko zwei parallele Gesprächsstränge, die sich gebildet haben, mit dem Verweis darauf, dass das „mit der kamera $n$ bisschen (.) EINfacher zu erkennen“ ist (Z. 404), wieder zusammenführen (Z. 398-405). Die anderen ratifizieren dieses Bestreben (Z. 405-407) und Stephanie weist Judith das Rederecht als nächstes zu (Z. 409). In einer anderen Gruppe entschuldigt sich Leni („SORry kamera;“, Anhang IV: MNG11, Z. 365), nachdem ebenfalls zwei synchrone Gespräche über metakommunikative Aufforderungen reorganisiert werden mussten (Z. 361-366).

Kooperativität bedeutet auf der anderen Seite auch, dass die Teilnehmenden die Aufgabe diskursiv und gemeinsam als Gruppe bearbeiten (vgl. auch Abschnitt 6.1.1). Daher kommt es so gut wie nie zu Schismen innerhalb der Gruppe bzw. diese werden schnell wieder aufgelöst ${ }^{3}$. Dass sich die Teilnehmenden an diesem Prinzip orientieren, bedeutet nicht, dass alle Gruppen jederzeit versuchen, einen Konsens zu etablieren. Meistens finden sich zwar Anzeichen für die Präferenz von Konsens - auch bei unterschiedlichen Bewertungen einer Serie - es gibt aber gleichermaßen Sequenzen, in denen Dissens mal eher face-bedrohend (vgl. Analyse von Beispiel 51), mal vergnüglich (vgl. Analysen in Abschnitt 7.3.3) verhandelt wird.

3 Hier deuten sich Parallelen zu digital vermittelter Kommunikation über Videokonferenzen mit mehreren Teilnehmenden an, bei denen auch - abseits der Möglichkeit, kleinere Gruppenräume zu betreten - in der Regel die Aufmerksamkeit aller auf der Hauptinteraktion liegt und der turnby-turn-Talk entsprechend in größerem, öffentlicherem Rahmen organisiert werden muss. 
Face-bedrohende Äußerungen finden sich jedoch eher selten in den Daten, was vermutlich (auch) auf die institutionelle und öffentliche Rahmung zurückzuführen ist.

Positionierungen können also nicht nur gegenüber den Interagierenden vorgenommen werden, sondern können sich auch an die Kamera und/oder die Forscherin richten. Besonders eindrücklich zeigt sich daran auch, dass sich die Jugendlichen gerade in Zeiten, in denen Videos sofort und jederzeit erstellt, gespeichert und digital verfügbar gemacht werden können, einer (potentiellen) Öffentlichkeit durch die Aufnahmesituation bewusst sind und ihre Positionierungen daran ausrichten können. Bisweilen spielen die Jugendlichen mit der Frage nach den Adressat ${ }^{\star}$ innen ihrer Äußerungen und inszenieren sich und einander, indem sie immer wieder metakommunikative und erklärende Kommentare in Bezug auf die Kamera und zur Kamera gewandt abgeben (vgl. dazu exemplarisch Anhang VII: RGM07, Z. 132-184). Für die Datenanalyse hat das insofern Konsequenzen, als die jeweiligen Kontextualisierungshinweise für die Interpretation hinzugezogen werden, wenn sie für die Analyse der Positionierungsaktivitäten und das Herausarbeiten der Praktiken relevant sind.

\subsection{Rekonstruktion der interaktiven Ziele}

\subsection{1 (Ermöglichen von) Partizipation}

In Abschnitt 2.2.3 wurde erläutert, dass Partizipation im Kontext von Medienkommunikation eine zentrale Rolle zukommt. Vor diesem Hintergrund lässt sich das in Abschnitt 6.1.2 beschriebene Prinzip der Kooperativität der Teilnehmenden mit dem Konzept der Partizipation analytisch erfassen. Mit Partizipation werden in Anlehnung an Goodwin und Goodwin (2006: 222) ,actions demonstrating forms of involvement performed by parties within evolving structures of talk" verstanden. Partizipation ist jedoch in den vorliegenden Daten nicht nur rein auf der Ebene der Interaktionsorganisation angesiedelt, sondern kann ebenfalls als Interaktionsziel gelten, da die Jugendlichen die Gespräche als diskursiv und möglichst von allen Teilnehmenden zu bearbeitende Aufgabe behandeln (Abschnitt 6.1). Das zeigt sich indirekt in der Art der Gesprächsorganisation (Beispiele 1 und 5) und an der häufigen Wahl des Personalpronomens in der ersten Person Plural in Vorschlags- oder Aufforderungsformulierungen zu Beginn der Gespräche „,̈̈ber so serien reden die wir so aktuell so GUCKen;“ (RHG10, Z. 006), „die wir alle kennen“ (EMS53, Z. 003, vgl. Beispiel 6). Expliziter kommunizieren diesbezüglich die jüngeren Teilnehmenden, beispielsweise wenn ein Gruppenmitglied verbal wenig partizipiert: „DAria; 
(.) du hast heute noch fast nichts geSAGT; “ (Anhang VII: RGM07, Z. 241). Auch in der folgenden Sequenz wird Partizipation als zu erreichendes Ziel explizit deutlich:

(6) ne serie die wir alle KENnen;

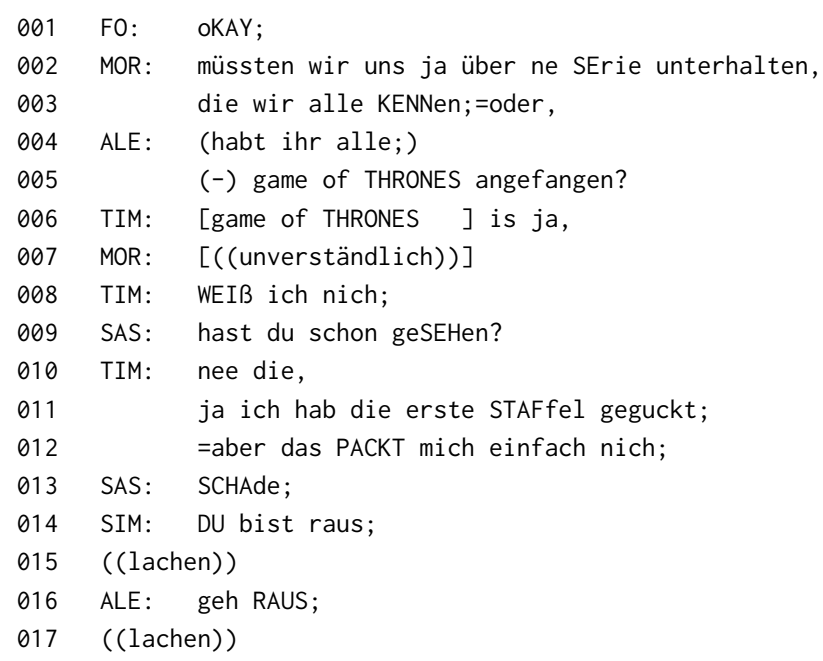

Moritz markiert in dieser Sequenz zu Beginn des Gesprächs metadiskursiv, dass die potentielle Beteiligung aller Teilnehmer erstrebenswert ist (Z. 002-003). Daraufhin adressiert Alex die Gruppe und fragt, ob alle die Serie GAME of THRONES kennen. Diese Serie wird von den meisten Teilnehmenden als eine mit hoher Wahrscheinlichkeit allgemein bekannte behandelt (vgl. auch Abschnitt 9.1). Darauf deuten auch Simons und Alex' Äußerungen hin, indem sie Tim, den die Serie nicht „packt“ (Z. 012)4, scherzhaft als „raus“ (Z. 015, 016) positionieren. „Raus“ bedient dabei mehrere Bedeutungsebenen: Zum einen eine räumliche Bedeutung im Wortsinn, da Alex währenddessen mit dem Daumen hinter sich auf die Tür deutet und zum anderen eine metaphorische Bedeutung, da Tim anzeigt, keinen Zugriff auf eine als geteilt angenommene Wissensgrundlage $\mathrm{zu}$ haben. Damit behandeln die Teilnehmer Tim ironisch-scherzhaft (Z. 015-017) als jemanden, der nicht die notwendigen Voraussetzungen für das Gespräch mitbringt und daher des gemeinsamen ,(Partizipations-)Raums“ verwiesen wird. Dass die Teilnehmenden meistens - eigene Gesprächspartizipation anstreben und anderen ermöglichen,

4 Vgl. zu diesem Aspekt auch Beispiele 27 und 48 und Analysen in den Abschnitten 8.1.1 und 8.1.3. 
bildet die Grundlage für die Analyse der Positionierungspraktiken in Abschnitt 7.1 und 7.2. Insbesondere schlägt sie sich in den Praktiken Managen divergenter Wissens(be)stände (Abschnitt 7.1.1) und Absprechen fremder epistemischer Autorität (Abschnitt 7.1.3) nieder.

\subsubsection{Sich mit anderen vergemeinschaften und voneinander abgrenzen}

Vergemeinschaftung und Distinktion können bisherigen Forschungsergebnissen zufolge sowohl als Ziele und interaktive Funktionen von Positionierungsaktivitäten gelten (vgl. Abschnitt 3.2.5) als auch als Ziele und interaktive Funktionen von Gesprächen über Medien (vgl. Abschnitt 2.2.3). In den vorliegenden Daten spielt das Austarieren und Changieren zwischen hergestellter Nähe und Distanz der Interagierenden durch Zustimmen und Widersprechen eine zentrale Rolle. Die Teilnehmenden wechseln also zwischen „,doing togetherness“ (Goebel 2013) und „doing being extraordinary“ (Sacher 2012) (vgl. Abschnitt 3.2.3), d.h. sie vergemeinschaften sich miteinander und grenzen sich voneinander $a b$.

Eine Sequenz, in der sich die gesamte Gruppe vergemeinschaftet, wird in Abschnitt 7.3.1 analysiert; meistens bilden sich jedoch kleinere Allianzen zwischen Subgruppen oder einzelne Teilnehmende distanzieren sich von anderen Teilnehmenden bzw. Subgruppen. So hält beispielsweise Jans lakonische Äußerung „die haben einfach KEInen geschmack; “(RHG10, Z. 641, vgl. Beispiel 32) sowohl die Vergemeinschaftung zwischen ihm und Leon als auch die Distinktion zwischen den beiden und den Teilnehmerinnen der Gruppe (die eine Serie, die Jan und Leon mögen, negativ bewerten) aufrecht. Auch Roberts Zusicherung an Johann „da bin ich VOLL auf deiner seite; “ (OST11, Z. 979; vgl. auch Analysen in Abschnitt 9.1.2) verfestigt zuvor in der Gruppe gebildete Allianzen. Distanzierung und die darüber etablierte Positionierung als individueller und unabhängiger Serienrezipient wird in folgender Sequenz deutlich:

(7) ich steh zu den serien die ich GUCK;

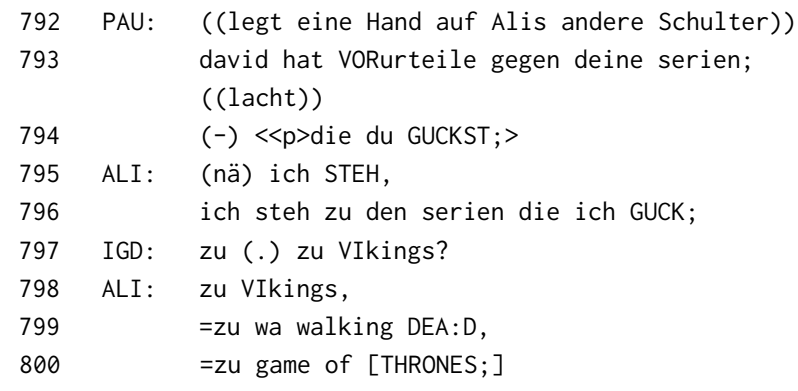




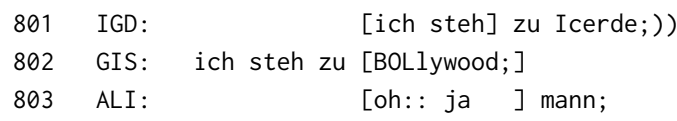

Die Sequenz ist eingebettet in das Fortführen von Frotzel-Aktivitäten (Günthner 2006) gegen Ende des Gesprächs, mit denen David durchgängig während der Interaktion Ali konfrontiert, da er die Serien, die Ali gefallen, negativ bewertet ${ }^{5}$. Paul steigt in die Scherzkommunikation ein, indem er eine Hand auf Alis Schulter legt und äußert: „david hat VORurteile gegen deine serien;“ (Z. 793). Ali stellt daraufhin explizite Distinktion her (Z. 795-800), indem er zu den Serien „steht“, die zuvor Gegenstand der Anfechtung seitens David waren. Damit löst er eine Art „Distinktions-Kaskade“ aus, da danach sowohl Igdir als auch Gisem seine Formulierung übernehmen (Z. 801, 802). Tatsächlich hat allerdings zuvor nur Gisem eine mit Ali vergleichbare Erfahrung gemacht, da sie ihre Präferenz für Bollywood sehr ausgiebig verteidigen muss (vgl. Analyse von Beispiel 22). Vor diesem Hintergrund können die Distinktionsaktivitäten von Igdir und Gisem auch wiederum als Vergemeinschaftungsangebot an Ali verstanden werden. Vergemeinschaftung und Distinktion als komplexe, interaktive Ziele, die eng mit dem Partizipationsbestreben zusammenhängen, bilden die Grundlage für die Analysen der Positionierungspraktiken in den Kapiteln 7 und 8.

\subsection{Rekonstruktion der kommunikativen Aufgaben}

Die Analyse bezieht ebenfalls ein, von welchen Möglichkeiten, auf den Gesprächsgegenstand interaktiv zu verweisen, die Teilnehmenden Gebrauch machen. Aus den bisher analysierten Beispielen lässt sich bereits erkennen, dass Positionierungen sich insbesondere in Form von Bewertungen von Serien (wie in Beispiel 7) manifestieren und wissensbezogene Aushandlungen von Serien (wie in Beispiel 6) begünstigen. Beides wird in Anlehnung an Hausendorfs Studien zur Kunstkommunikation (vgl. u.a. Hausendorf 2007, 2012, 2016 und Abschnitt 2.2.2 sowie 2.2.3) als kommunikative Aufgaben verstanden, d.h. als „Antworten auf Fragen, [...] die es kommunikativ zu bearbeiten gilt“ (Hausendorf 2012: 99). Die Aufgaben, die die Teilnehmenden in den vorliegenden Gesprächsdaten als Antworten auf „zugrunde liegende Fragen“ bearbeiten - die sich aus der Instruktion und der Kontextualisierung des Gesprächstyps ergeben -, lassen sich empirisch rekonstruieren als

5 Vgl. dazu auch die Analysen zu Beispiel 17 und in den Abschnitten 8.1.4 und 9.1.1. 
Bewerten und Wissen anzeigen. Sie sind angelehnt an die beiden Aufgaben „Bewerten (Was ist davon zu halten?)“ und „Erläutern (Was weiß man darüber)?“ (Hausendorf 2011: 524), die Hausendorf im Kontext von Kunstkommunikation identifiziert, unterscheiden sich jedoch aufgrund ihrer empirischen Fundierung von Hausendorfs Befunden folgendermaßen:

- Bewerten - beantwortet die Frage danach, wie die Teilnehmenden konkrete Serienproduktionen bzw. Serien als narrativ und ästhetisch gestaltete Medienprodukte auf einer abstrakteren Ebene und/oder serienrezeptionsbezogene Aspekte in positiver oder negativer Weise bewerten.

- Wissen anzeigen - beantwortet die Frage danach, wie gut die Teilnehmenden konkrete Serienproduktionen bzw. Serien als narrativ und ästhetisch gestaltete Medienprodukte und/oder serienrezeptionsbezogene Aspekte kennen sowie welche Wissenselemente ihnen bekannt sind und wie viel sie (nicht) wissen (wollen).

Diese Verweisvarianten sind nicht dichotom aufzufassen, sondern werden immer wieder in ihrem Zusammenspiel und ihrer Funktion für die Herstellung von Vergemeinschaftung und Distinktion analysiert. In der Regel werden Elaborierungen und Begründungen, die über minimale evaluative oder epistemische Displays (i.S.v. „das finde ich gut“ oder „das kenne ich auch“) hinausgehen, vorgenommen bzw. bei Ausbleiben eingefordert. Im Folgenden werden beide kommunikativen Aufgaben zunächst separat anhand einer fortlaufenden Gesprächssequenz näher erläutert, bevor in Abschnitt 6.4 auf deren Zusammenhang eingegangen wird.

\subsubsection{Bewerten}

Mit der Instruktion der Forscherin, sich über Serien zu unterhalten, die die Jugendlichen gerne schauen, ist die kommunikative Aufgabe des Bewertens schon implizit angelegt. Deutlich wird das auch an Alis Formulierung in Beispiel 7, er würde zu den von ihm favorisierten Serien „stehen“ (MNG13, Z. 796). Anhand der folgenden Sequenz zu der Serie ScRUBS wird weiterführend herausgearbeitet, wie Bewertungen für die in Abschnitt 6.2 angeführten interaktiven Ziele funktionalisiert werden.

(8) oh ich LIEbe es;

176 BIL: aber,

177 (.) in letzter zeit guck ich auch eine ALte serie die gar nich mehr läUft; 


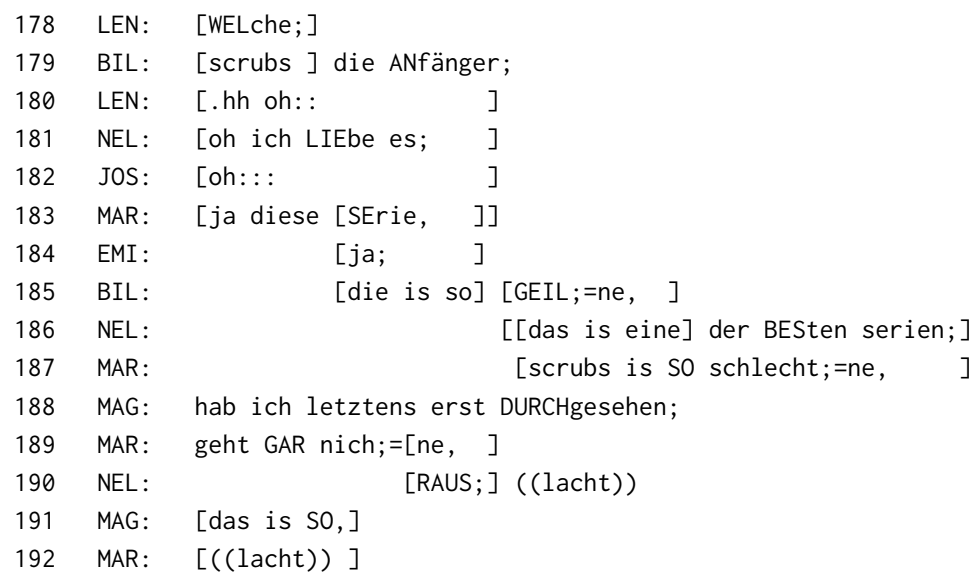

Bilel nennt zunächst nicht den Titel, sondern kündigt die Serie an als eine „ALte serie die gar nicht mehr lÄuft;“" (Z. 177). Auf seine Präzisierung hin, dass es sich dabei um SCRUBS handelt, folgen viele parallele Äußerungen in Form von ,extreme case-Formulierungen“ (Pomerantz 1986) (Z. 181, 186) und Interjektionen (Z. 180, 182), die eine positive Bewertung ausdrücken. Bilel kommuniziert während dieser Bewertungskaskaden ebenfalls seine sehr positive Haltung gegenüber der Serie: „die is so GEIL; =ne,“ (Z. 185). Bilel, Leni, Emilia und Nelli vergemeinschaften sich, indem sie sich in ihrer Bewertung wechselseitig bestätigen und zustimmen. Martha zeigt in dieser Sequenz als einzige Teilnehmerin eine negative Haltung gegenüber der Serie mit einer Äußerung an, die zwar syntaktisch mit Bilels Äußerung aligniert ist, aber keine Zustimmung aufweist: „scrubs is SO schlecht;=ne,“(Z. 187). Damit signalisiert sie ähnlich wie Tim in Beispiel 6 Distinktion von der Mehrheit der Gruppe (siehe auch Z. 189). Die Folgebehandlung der Gruppe ist ebenfalls mit diesem Fall vergleichbar, da Martha aufgrund ihrer negativen Bewertung - scherzhaft als „raus“ (Z. 190) positioniert wird.

Bewerten als kommunikative Aufgabe bietet also Möglichkeiten zur Etablierung von Vergemeinschaftung bzw. Distinktion, aber auch für das Ziel der Partizipation. Denn nachdem Bilel mit ScRUBS eine Serie genannt hat, die er gerne schaut, ist es für die anderen notwendig, sich ebenfalls dazu zu positionieren, um an der folgenden Sequenz partizipieren zu können. Über zustimmende Bewertungen, wie sie Nelli, Emilia und Leni produzieren, beteiligen sie sich an der Interaktion im Sinne der Instruktion. Martha partizipiert zwar ebenfalls, indem sie eine Bewertung äußert, doch da diese Bewertung nicht von den anderen geteilt wird, hat sie Schwierigkeiten, zu partizipieren (vgl. Z. 190): So minimieren sich 
tatsächlich ihre Redebeiträge im Laufe der ScRUBS-Sequenz im Kontrast zu den vorherigen und nachfolgenden Sequenzen. In dieser Studie werden somit evaluative Positionierungen der Teilnehmenden analysiert, die sich in einem Kontinuum zwischen negativ über neutral bis positiv befinden und über die per Zweitbewertung Zustimmung oder Widerspruch ausgedrückt werden kann (vgl. auch Abschnitt 4.3). Für Serienkommunikation ist Bewerten in hohem Maße von Bedeutung, da Serien als ästhetisch gestaltete Erzeugnisse eine subjektive Beurteilung bereits nahelegen (vgl. Abschnitt 2.2.3).

\subsubsection{Wissen anzeigen}

Aus der Analyse von Beispiel 8 kann geschlossen werden, dass sowohl für Gesprächspartizipation als auch für Bewertungen eine grundlegende Kenntnis des Gesprächsgegenstands die Voraussetzung darstellt (vgl. dazu auch Pomerantz 1984). Anders gesagt: Wenn Martha nicht wüsste, um was für eine Serie es sich bei SCRUBS handelt, wäre es unwahrscheinlich, dass sie ihre Bewertung mit einer entsprechend hohen epistemischen Gewissheit (,scrubs is SO schlecht;=ne,“, Z. 185) ausgestattet hätte.

Es ist also in der Regel notwendig, dass sich serienbezogene Wissenselemente im common ground (Abschnitt 4.3) der Interagierenden befinden. In Anlehnung an Heritages (2012, 2012) Terminologie können die Teilnehmenden über ihre epistemische Haltung einander signalisieren, dass sie graduell über mehr Wissen als die anderen verfügen - dann ist ihr epistemischer Status $K^{+}$einzustufen - oder weniger wissen $\left(\mathrm{K}^{-}\right)$, wobei das Wissen wiederum hinsichtlich seiner Tiefe bzw. Oberflächlichkeit variieren kann. Bezogen auf den Gesprächsgegenstand kann sich das Wissen der Beteiligten zu einer spezifischen Serie also graduell von keiner Kenntnis des Titels über Hören-Sagen bis zu detailliertem Hintergrund-Wissen erstrecken; analog zu Kepplers (1994: 241) Unterscheidung zwischen „PrimärWissenden“, Personen, die über (Medien-)Wissen aus zweiter Hand verfügen, und solchen, die den rekonstruierten Inhalt überhaupt nicht kennen (vgl. Abschnitt 2.2.3). Das wird anhand der Fortsetzung zur ScRUBS-Sequenz in Beispiel 8 im Folgenden erläutert:

(9) die LETZte folge is so süß,

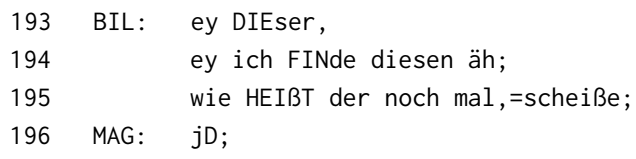




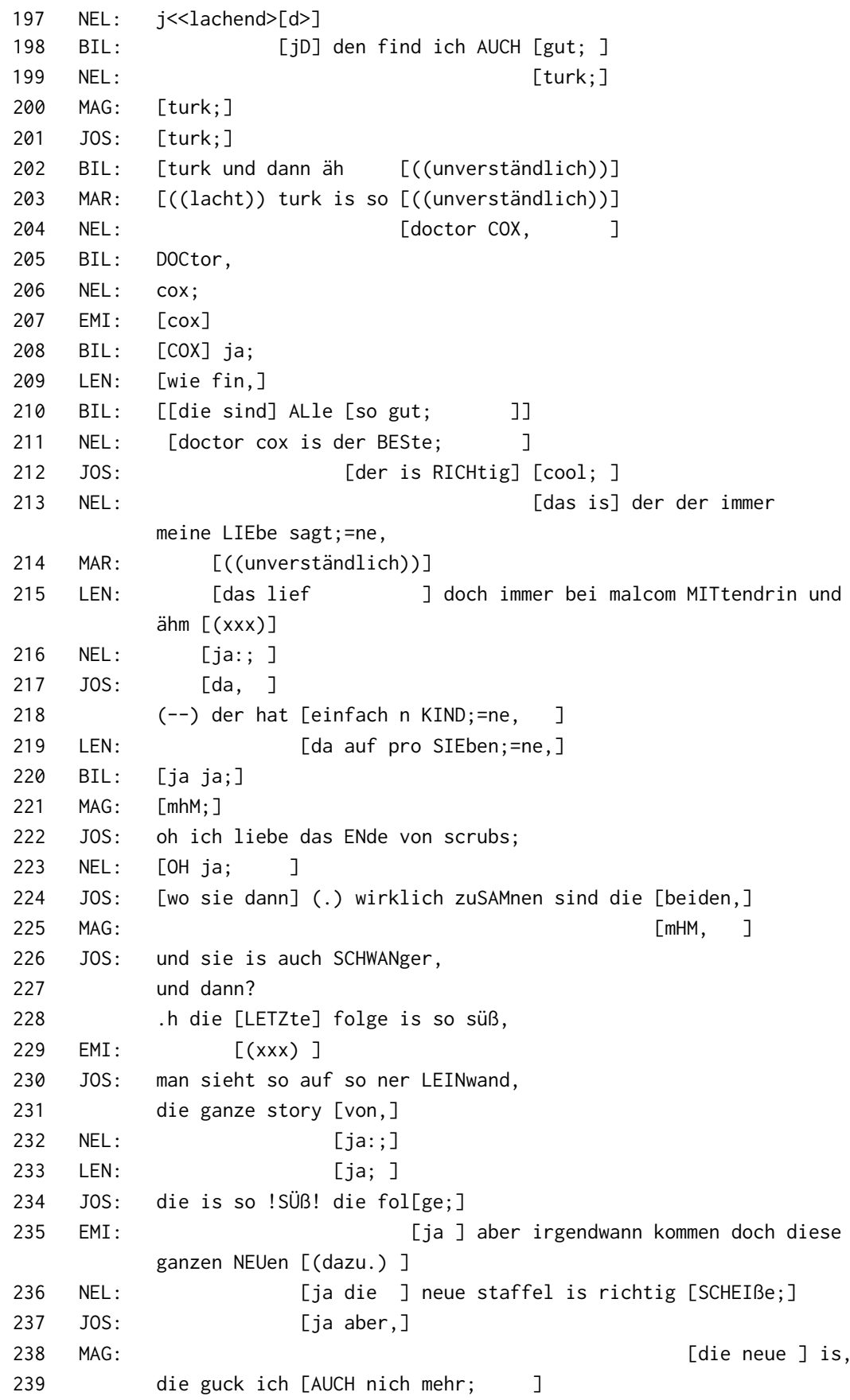




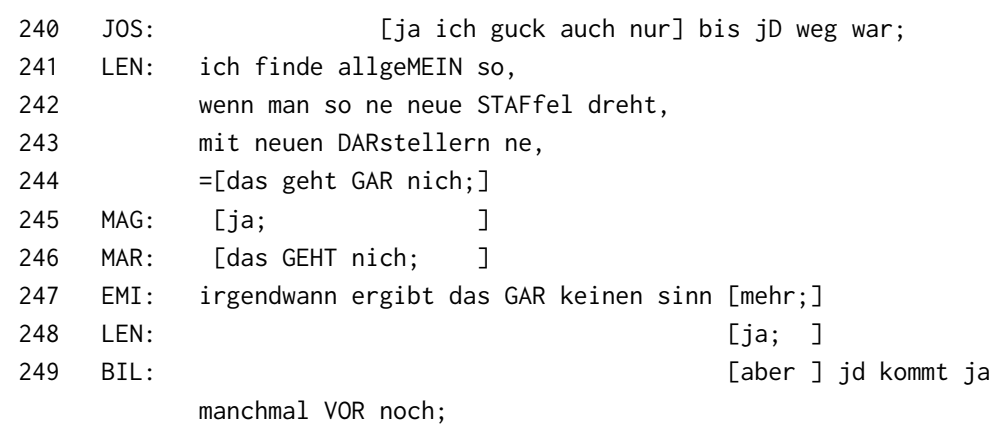

MNG11, 3:24-4:07

Neben weiteren Bewertungen der Figuren (Z. 103-213), der Qualität einzelner Staffeln (Z. 235-240) und Episoden (Z. 222-234) spielt hier das Anzeigen von (ausreichendem) Wissen zur Partizipation und Vergemeinschaftung sowie Distinktion eine Rolle. Es beginnt damit, dass Bilel den Namen einer Figur sucht (Z. 195), woraufhin ihm zwei Teilnehmende eine „candidate answer“ (Pomerantz 2009) anbieten (Z. 196-197), die Bilel ratifiziert. Dann folgen diverse überlappende Äußerungen, in denen Magnus, Josefine, Bilel, Nelli und Emilia mehrere Figuren aufzählen und einander in ihrer Bewertung diesbezüglich bestätigen. Die mehrfache Nennung der Figurennamen ${ }^{6}$ seitens fast jedes Gruppenmitglieds erfüllt mehrere Funktionen: Auf den ersten Blick wird dadurch Bilels eingangs angezeigte Wissenslücke behoben. Dadurch zeigen die Jugendlichen aber auch an, über einen geteilten common ground die Serie betreffend zu verfügen, wodurch sie einerseits Partizipationsbereitschaft signalisieren sowie sich gegenseitig Partizipation ermöglichen, und sie sich andererseits zugleich über ihr geteiltes Wissen (als Grundlage für eine geteilte Bewertung) miteinander vergemeinschaften.

Bei genauerer Betrachtung wird deutlich, dass die Teilnehmenden im Laufe dieser Sequenz unterschiedliche Arten von Wissenselementen in die Interaktion einbringen und darüber einen unterschiedlichen epistemischen Zugang zu der Serie kommunizieren. Während Bilel die Bewertung der Hauptfiguren initiiert, verweist Leni auf den Distributionskanal und -kontext der Serie (Z. 215-219) und Josefine gibt Detail-Wissen zur Bewertung preis, das sich auf konkrete Handlungselemente der Serie bezieht (Z. 222-234). Die Angabe, dass es sich um das „ENde“ (Z. 222) bzw. die „LETZte folge“ (Z. 228) handelt, gibt zudem einen Hinweis darauf, dass Josefine die komplette Serie kennt (wenn man von linearen Rezeptionsgewohnheiten ausgeht). Ihre epistemische Haltung signalisiert daher zum einen

6 Zur Bedeutung der expliziten Nennung von Figurennamen vgl. Abschnitt 8.1.4. 
einen fundierten epistemischen Zugang zu der Serie und zum anderen eine hohe epistemische Gewissheit. Dadurch beansprucht sie das epistemische Recht zur Evaluation. Ihre Bewertung wird interaktiv ratifiziert (Z. 225, 232-233), so dass ihr ebenfalls die epistemische Autorität zur Bewertung zugeschrieben wird und sie sich als Expertin positionieren kann (vgl. Ausführungen und Definitionen der Begriffe in Abschnitt 4.3). Anschließend initiiert Emilia eine heruntergestufte Bewertung der Serie, die implizit ebenfalls auf einen hohen epistemischen Zugang schließen lässt, so dass nun sie das epistemische Recht zur Evaluation beansprucht und dadurch, dass sich die anderen ihrer Bewertung zustimmend anschließen, ebenfalls als (kritische) Expertin für die Serie positioniert wird. Martha - die sich als einzige gegen die Serie positioniert (Beispiel 8), - äußert nur eine kurze Bewertung zu der Figur Turk (Z. 203) und partizipiert erst dann wieder am Gespräch, als das Thema auf eine abstraktere Ebene verschoben wird. Sie beteiligt sich zustimmend an der nachfolgenden Interaktion zu Lenis Aussage, dass Serien an Qualität verlieren würden, wenn Staffeln mit neuen Schauspieler^innen gedreht werden (Z. 241-248; vgl. zu diesem Aspekt auch Abschnitt 8.1.4).

Da Wissenskommunikation für die vorliegenden Gesprächsdaten als kommunikative Aufgabe betrachtet werden kann, finden sich auch keine komplexen Einstiege in Belehrungen, wie sie von Keppler (1989) beschrieben werden (vgl. dazu etwa die Analyse von Beispiel 25). Zusammenfassend werden in dieser Arbeit epistemische Positionierungen der Teilnehmenden analysiert, die sich in einem Kontinuum zwischen einem niedrigen bis zu einem hohen epistemischen Status befinden. Ein Ziel der Analyse besteht darin, Praktiken herauszuarbeiten, mit denen die Teilnehmenden - wie Josefine hier für SCRUBS - epistemische Autorität zur Bewertung beanspruchen (vgl. Abschnitt 4.3) und sich damit als Expertinnen positionieren (Kapitel 7). Wie in Abschnitt 2.2.3 diskutiert, spielt für Serienkommunikation - im Unterschied zu anderen Formen der Medienkommunikation die Variabilität des common ground eine zentrale Rolle: Serien bieten hochgradig individualisierte Möglichkeiten zur Rezeption, so dass die Teilnehmenden nie sicher sein können, wie viele Episoden oder gar Staffeln einer Serie die anderen Gruppenmitglieder gesehen haben. Zudem können sie auch nicht davon ausgehen, dass alle das Gesehene ähnlich interpretieren. Um dennoch das Ziel der Gesprächspartizipation zu erreichen, ist es einerseits notwendig, epistemische Asymmetrien abzubauen (Abschnitt 7.1.1), andererseits aber auch niemanden zu spoilern (Abschnitt 8.2.3). 


\subsection{Zum Zusammenhang zwischen Wissen und Bewerten im Rahmen von Serienkommunikation: (un-)wissende Unterstützer*innen und Kritiker*innen}

Um die in den vorherigen Abschnitten dargestellten Prozesse der Vergemeinschaftung und Distinktion zu erfassen, wurde eine Visualisierungshilfe entwickelt, die es im Rahmen des erarbeiteten Modells erlaubt, den Zusammenhang zwischen epistemischen und evaluativen Positionierungen innerhalb einer Sequenz schematisch abzubilden. Es wird davon ausgegangen, dass sich die Teilnehmenden in Bezug auf eine konkrete Serie ${ }^{7}$ epistemisch und evaluativ in einem ,Feld' positionieren, das in Form eines zweidimensionalen Koordinatensystems dargestellt werden kann. Auf der X-Achse wird eine Person über ihren rekonstruierten epistemischen Status (zwischen $\mathrm{K}^{+}$und $\mathrm{K}^{-}$) und auf der Y-Achse über ihre angezeigte Bewertung (positiv/negativ) bezogen auf eine Serie eingeordnet. Abbildung 6.1 verdeutlicht diesen Zusammenhang:

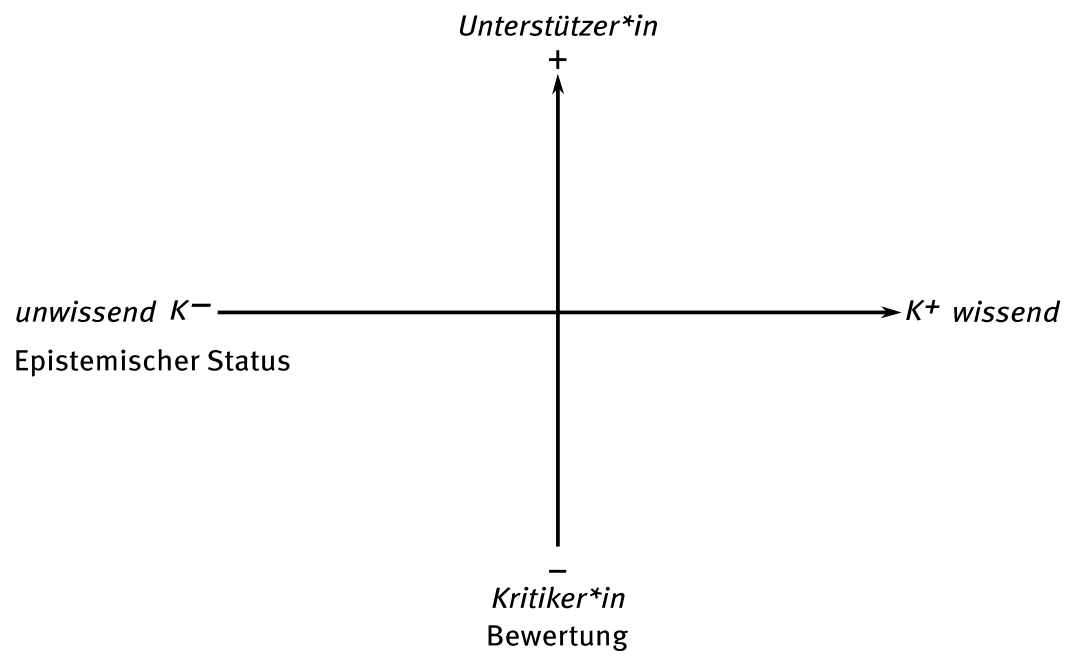

Abb. 6.1: Koordinatensystem zur relativen Bestimmung epistemischer und evaluativer Positionierungen von Interagierenden.

7 Vgl. zur Unterscheidung zwischen konkreten und abstrakten Positionierungsobjekten Abschnitt 6.5.1. 
Wendet man dieses Schema auf die Sequenz zu Scrubs an, wie sie in den Abschnitten 6.3.1 und 6.3.2 analysiert wurde, so ergeben sich folgende Konstellationen im zeitlichen Verlauf (Abbildung 6.2): Alle Gruppenmitglieder außer Martha zeigen zu Beginn der Sequenz, dass sie die Serie kennen und mögen, daher sind sie im oberen rechten Quadranten als wissende Unterstützer`innen eingeordnet, wobei die Einordnung keine absoluten Positionen suggerieren soll. Martha ist die einzige Teilnehmerin, die die Serie zwar kennt, sie aber negativ bewertet - daher kann sie in dem unteren rechten Quadranten verortet werden. Die Linien um die Sprecher*innenkürzel zeigen in Form von drei Abstufungen die Intensität der geäußerten Haltung an (vgl. zur Unterscheidung zwischen Intensität und Explizitheit Abschnitt 3.3, Merkmal (6)). So kommuniziert Magnus seine Haltung gegenüber der Serie - verglichen mit den anderen Beteiligten - am wenigsten intensiv (Z. 188). Im Verlauf der Sequenz verschiebt Nelli ihre Haltung, indem sie äußert, dass sie die neueren Staffeln schlechter findet als die älteren. Sie erhält Zustimmung von Josefine, Emilia, Magnus und Leni, die ihr daher in der Änderung ihrer Haltung gegenüber der Serie folgen; lediglich Bilel bleibt demgegenüber indifferent. Diese Veränderung wird in der zweiten Abbildung dergestalt visualisiert, dass die Teilnehmenden sich zum Ende der Sequenz eher als wissende Kritiker^innen der Serie positionieren - wobei diese Positionierung eine punktuelle Relation zu den anderen Interagierenden abbildet. Insgesamt ergibt sich die Perspektive der Visualisierung aus der sequenziellen Analyse des Gesprächsausschnitts heraus, sodass darin retrospektiv schematisch Prozesse innerhalb der Sequenz abgebildet werden.

Der Mehrwert dieser schematischen Darstellung liegt darin, Analyseergebnisse einzelner Sequenzen nicht nur auf einen Blick sichtbar zu machen, sondern auch Fälle systematisch auf diesen Aspekt hin miteinander vergleichen zu können. Dabei muss natürlich bedacht werden, dass es sich - wie bei allen Formen der visuellen Aufbereitung von Datenmaterial im qualitativen Forschungsparadigma - um interpretative Konstrukte seitens der Forscher`innen handelt. Im Laufe der Analysen in Kapitel 7 wird punktuell auf diese Form der Visualisierung zurückgegriffen, um die Positionierungspraktiken der Teilnehmenden darzustellen; in Kapitel 9 wird der Zusammenhang zu den Analyseergebnissen in Kapitel 8 hergestellt. 

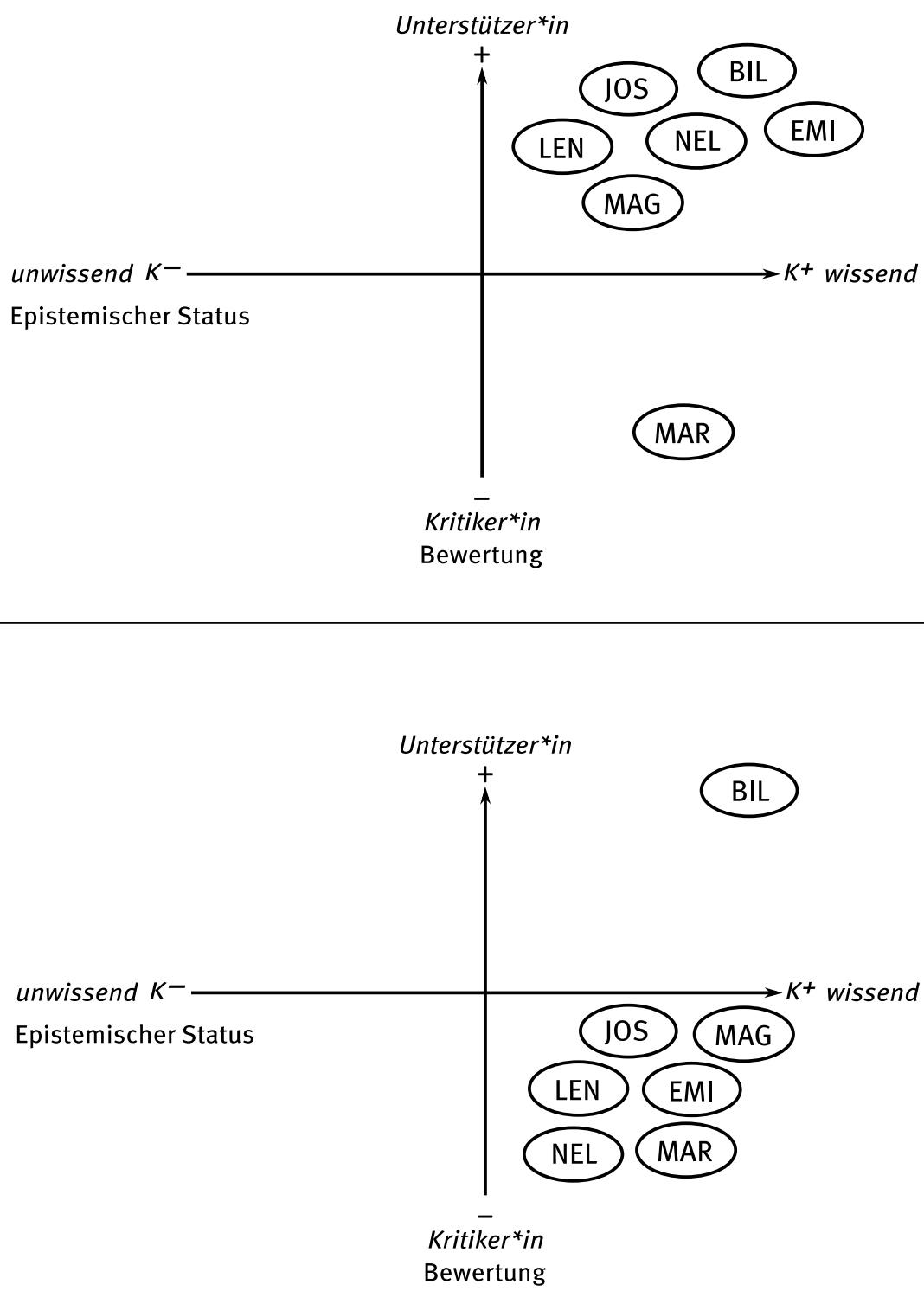

Abb. 6.2: Oben: Koordinatensystem zur relativen Bestimmung epistemischer und evaluativer Positionierungen der Interagierenden in Bezug auf die Serie SCRUBS zu Beginn der Sequenz. Unten: Veränderungen der relativen Positionierungen am Ende der Sequenz. Die Stärke der Linien um die Sprecher*innensiglen gibt die relative Intensität der Positionierungen an. 
Insgesamt kann bisher für die Analyse von Positionierungen festgehalten werden, dass die Teilnehmenden die Interaktionssituation folgendermaßen gestalten: Sie behandeln die Instruktion als eine gemeinsam und diskursiv zu bearbeitende Aufgabe unter den kontextuellen Bedingungen von Institutionalität und Öffentlichkeit, wobei sie dem Prinzip der Kooperativität (der Forscherin gegenüber und untereinander) folgen. Das heißt, sie gestalten das Gespräch interaktiv und relational, indem sie sowohl selbst daran partizipieren als auch anderen Personen Partizipation ermöglichen. So stellen sie punktuell und dynamisch Gemeinschaft und Abgrenzung her, indem sie eine epistemische und/oder evaluative Haltung gegenüber dem Gesprächsgegenstand kommunizieren, die sich im Laufe der Sequenz verändern kann. Damit werden die Merkmale von Positionierungen (1) Kontextgebundenheit, (2) Interaktivität und Relationalität sowie (3) Dynamik (vgl. Ausführungen in Abschnitt 3.3) berücksichtigt. Merkmal (4) der Gegenstandsbezogenheit wird im Folgenden weiter ausdifferenziert.

\subsection{Umgang mit dem Gesprächsgegenstand}

Im Folgenden werden Serien als Gesprächsgegenstand in den Blick genommen. Dafür wird zunächst herausgearbeitet, welche thematischen Aspekte die Teilnehmenden in den Gruppendiskussionen relevant setzen (Abschnitt 6.5.1). Anschließend wird erläutert, welche Ressourcen die Jugendlichen verwenden, um sich in Bezug auf den Gegenstand zu positionieren (Abschnitt 6.5.2). Diese Ressourcen werden in Anlehnung an die Formen von Positionierungen in Abschnitt 3.2 beschrieben. Abschließend wird die Funktion des Gesprächsgegenstands als sequenzstrukturierendes Mittel dargestellt (Abschnitt 6.5.3) sowie erläutert, welche Konsequenzen sich daraus für die Analyse ergeben.

\subsubsection{Das Positionierungsobjekt - interaktiv relevant gesetzte Aspekte des Gegenstands}

Der thematische Rahmen der Gespräche ist zwar durch die Instruktion vorgegeben (vgl. Abschnitt 6.1.1), die weitere inhaltliche Ausgestaltung in Form von Subthemen ist jedoch den Teilnehmenden überlassen. So wird in Anlehnung an Du Bois' stance object (vgl. Abschnitt 3.2.4.3) in dieser Arbeit der Gesprächsgegenstand Serien als ästhetisches und narratives Erzeugnis (vgl. Kapitel 2) - als Positionierungsobjekt definiert. Serien bilden den zugrundeliegenden Referenzrahmen für die Interaktion, auf den die Teilnehmenden die meiste Zeit über Bezug nehmen und an dem sie sich gesprächsstrukturell orientieren. Es wird in dieser Studie 
empirisch rekonstruiert, welche Aspekte des Positionierungsobjekts die Teilnehmenden durch epistemische und evaluative Bezugnahmen in welcher Art und Weise mit Bedeutung versehen. Damit beschränkt sich der analytische Fokus auf die erste der von Kiesling (2016) beschriebenen Dimensionen - diskursive Repräsentationen (vgl. Abschnitt 3.2.4.3) -; metakommunikative Äußerungen werden nicht berücksichtigt und Interagierende, denen der Platz des Objekts zugewiesen wird, nur punktuell analytisch einbezogen.

Das Positionierungsobjekt kann vielfältig ausgestaltet sein. Häufig besteht es aus einer konkreten Serie, wie in Beispiel 8 (SCRUBS) oder 12 (ADVENTURE TIME). Für das Sprechen über konkrete Serien ist für die Partizipation und Vergemeinschaftung bzw. Distinktion relevant, was und wie viel die Interagierenden darüber wissen und wie sie die Serie davon ausgehend bewerten. So können sie über ihre entsprechend empirisch rekonstruierten epistemischen und evaluativen Haltungen in das in Abschnitt 6.4 skizzierte Koordinatensystem eingeordnet werden. Konkrete Positionierungsobjekte werden primär in Kapitel 7 unter dem Begriff der lokalen Reichweite von Positionierungen analysiert. Positionierungsobjekte können aber auch abstrakter ausfallen und sich in Diskussionen über Serienformen (wie in Beispiel 11), Serialität (Abschnitt 9.1.1) oder Serienrezeption (Beispiel 52) manifestieren. Partizipation und Vergemeinschaftung bzw. Distinktion werden dann über diesbezüglich (nicht) geteiltes Wissen oder Bewertungen erreicht. Geteiltes Wissen bzw. geteilte Bewertungen beim Sprechen über abstrakte Positionierungsobjekte sind - überwiegend - nicht auf die Rezeption einer konkreten Serie (oder Serien generell) angewiesen, sie erfordern also keinen epistemischen Zugang zu einem spezifischen Medienprodukt. So kann sich beispielsweise Martha zu dem abstrakteren Sachverhalt - neue Staffeln einer Serie werden mit wechselnden Schauspieler`innen gedreht - positionieren, auch ohne dass sie eine gerade diskutierte Serie (gut) kennt (vgl. Beispiel 9). Stattdessen spielen vor allem kulturell geteilte und sozial erworbene und erlernte Wissensdomänen sowie Wertungen eine Rolle für Positionierungen. Aus diesem Grund werden solche Positionierungen, die in Kapitel 8 analysiert werden, als Positionierungen mit globaler Reichweite bezeichnet.

Grundlegend kann beim Positionierungsobjekt nicht nur zwischen konkreten und abstrakten Objekten unterschieden werden, sondern auch, ob die Teilnehmenden eher medien- oder eher rezeptionsbezogene Aspekte thematisieren. Medienbezogene Aspekte verweisen auf Serien als gestaltete Produkte und rezeptionsbezogene Aspekte auf die rezipient*innenseitige Wahrnehmung von Serien als gestaltete Produkte. Diese Unterscheidung ist empirisch deswegen relevant, weil sich unterschiedliche diskursive Orientierungen - prototypisch bzw. normativ der Teilnehmenden in Bezug auf diesen Dimensionen zugrundeliegende Wissensdomänen und Wertungen rekonstruieren lassen (vgl. Abschnitt 8.1 und 8.2). 


\subsubsection{Gegenstandsbezogene Ressourcen zur Positionierung}

Als Ressourcen werden die folgenden Aktivitäten verstanden, mit denen die Teilnehmenden ihre Haltung gegenüber einem Gegenstand anzeigen, wodurch sie sich und andere positionieren können. Die Ressourcen wurden analytisch in Anlehnung an die in Abschnitt 3.2 beschriebenen Varianten der Positionierungsforschung (Kategorisieren, Kontrastieren, narrative und biographische Identitätsforschung) von Positionierungen rekonstruiert:

- Kategorisieren und Zuordnen

- Kontrastieren und Vergleichen

- Rekonstruieren und Inszenieren

\subsubsection{Kategorisieren und Zuordnen}

Die Beschreibung dieser Ressource orientiert sich an den in Abschnitt 3.2.2 dargelegten Grundlagen zum Zusammenhang von Kategorisieren und Positionieren. Damit werden kategoriale Zuordnungen oder Zuschreibungen zu einer Serie oder einer Person erfasst, wobei die meisten Zuordnungen sich auf Serien und Serientypen beziehen (siehe dazu Analysen in Abschnitt 8.1.2), während nominale Personenreferenzen eher selten zu finden sind (siehe dazu Abschnitt 8.2.4.2).

Kategorisieren als Ressource für Positionierungen kann vielfältig funktionalisiert werden. Meistens wird sie dafür genutzt, sich einer geteilten Bedeutung $\mathrm{zu}$ vergewissern und wechselseitig Verständnis zu sichern (vgl. dazu auch die Analysen in Abschnitt 9.1.1). Das zeigt sich exemplarisch in dem folgenden Beispiel, in dem eine Serie der Kategorie case of the week zugeordnet wird:

(10) case of the WEEK (.) folge,

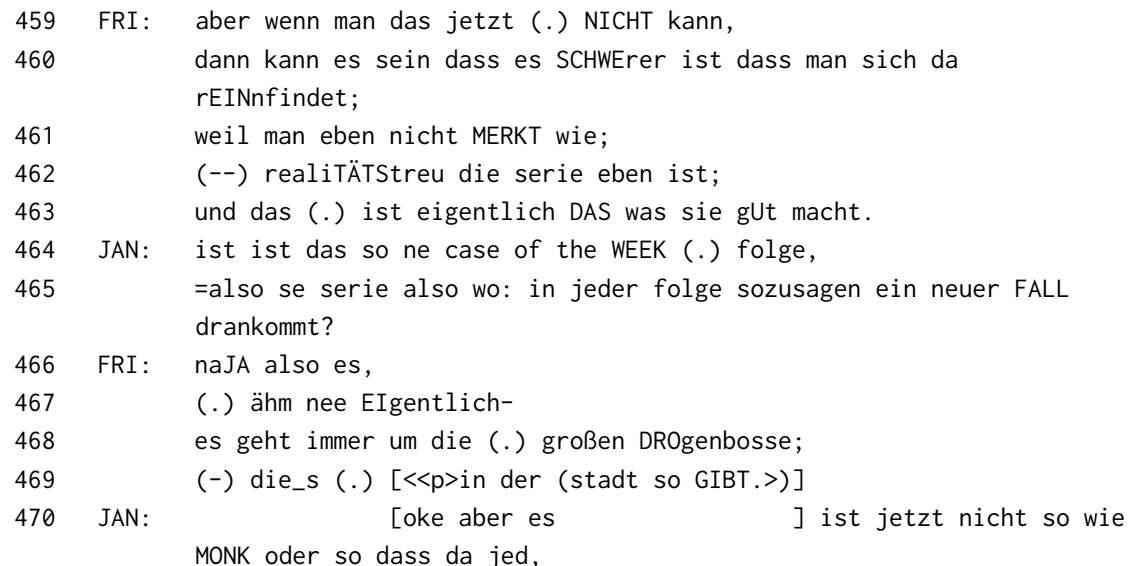




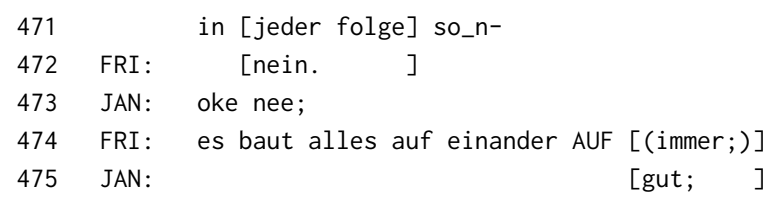

Die Sequenz ist eingebettet in Friederikes Erläuterungen zu der Serie THE WIRE, die die anderen Gruppenmitglieder nicht kennen (Z. 459-463; vgl. dazu auch Analysen zu Beispiel 19). Jan stellt Friederike eine Nachfrage zur Erzählweise der Serie (Z. 464-465) und weist ihr damit das Rederecht weiterhin zu. Dadurch sorgt er auch dafür, dass sowohl er als auch sie am Gespräch partizipieren können, und agiert im Sinne des Kooperativitätsprinzips, in dem er ihr und der von ihr genannten Serie Gesprächsraum überlässt. Er kategorisiert die Serie als „case of the $W E E W^{\prime \prime}$ (Z. 464) und elaboriert im nächsten Turn die Bedeutung dieser Kategorie: Es handelt sich um eine Serie, die sich jede Woche einem neuen Kriminalfall widmet (Z. 465). Damit stellt er zwar eine Informationsfrage an Friederike und weist ihr den relativ gesehen höheren $\mathrm{K}^{+}$-Status zu, signalisiert aber zugleich auch partielles Wissen über serielle Formen: Er greift auf das für Kriminalserien typische narrative Gestaltungsprinzip zurück und wendet es auf ihm unbekannte Serien an. Friederike weist seine Kategorie jedoch höflich zurück (Z.466-469), woraufhin Jan erneut - dieses Mal mittels eines Vergleichs (Abschnitt 6.5.2.2) - ein geteiltes Verständnis der Einordnung der Serie sichert (Z. 470-475).

\subsubsection{Kontrastieren und Vergleichen}

Kontrastieren wird in Anlehnung an die Grundlagen in Abschnitt 3.2.3 als eng mit Kategorisieren verknüpft verstanden, da die Teilnehmenden häufig Kategorien miteinander vergleichen. Die Ressource umfasst daher Vergleiche auf einer Ebene, wobei zwei Elemente in ein dichotomes Verhältnis gesetzt werden. Diese Elemente können intraseriell (d.h. innerhalb einer Serie; Beispiel 15), interseriell (zwischen zwei Serien; Beispiel 19) oder transseriell (serienübergreifend; Beispiel 11) vorgenommen werden sowie auf das eigene damit verbundene Rezeptionsverhalten und/oder -erleben bezogen werden. Oft entstehen daraus dann evaluative Positionierungen, indem eine stärkere Affinität zu einem der Elemente gezeigt wird. Kontrastieren kann ebenfalls zum Aufrechterhalten von Dissens genutzt werden (vgl. für eine ausführliche Analyse Abschnitt 7.3.3 oder Beispiel 50 in Abschnitt 8.2.4.3). Exemplarisch für das Einsetzen dieser Ressource wird folgende Sequenz analysiert: 
(11) es gibt ZWEI arten von serien;

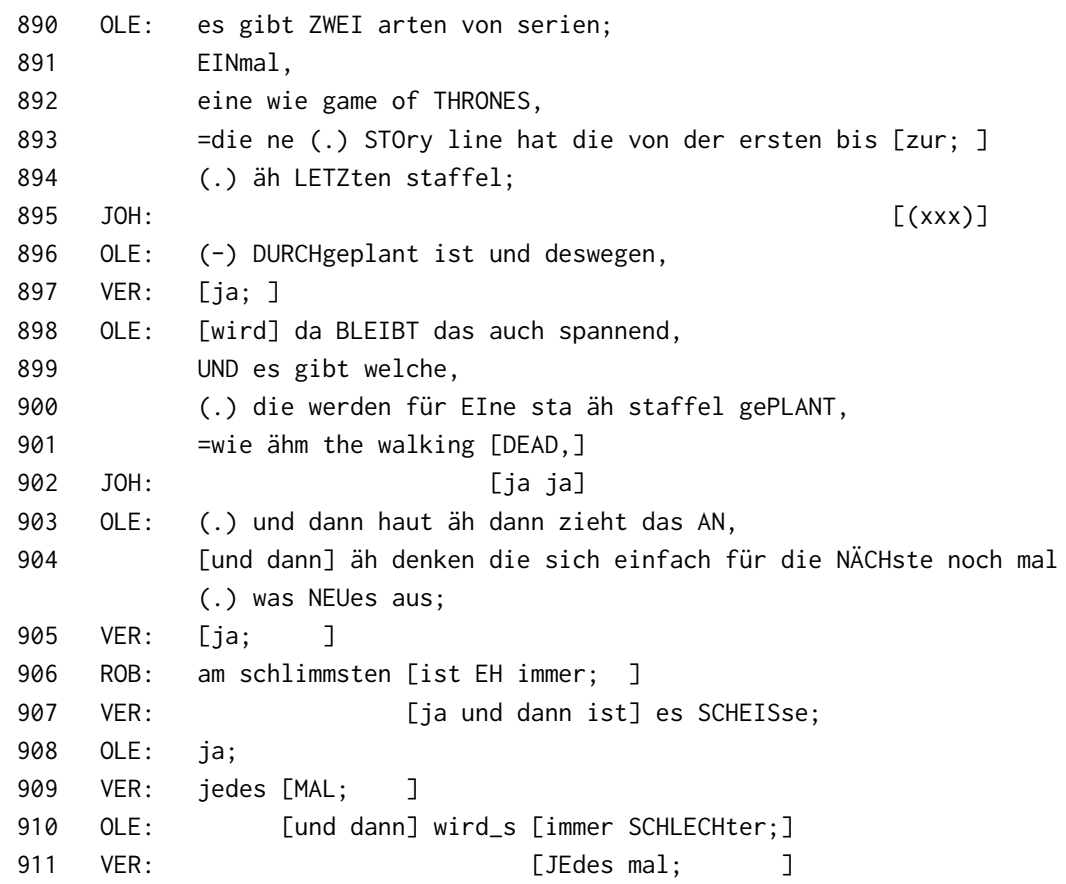

Diese Sequenz schließt direkt an die Diskussion zu (Un-)logik in Superheldenserien an (vgl. Beispiel 35). Ole kontextualisiert daran anknüpfend metakommunikativ einen transseriellen Kontrast, indem er „ZWEI arten von serien;“ (Z. 890) postuliert, die sich diametral gegenüber stehen. Die erste Art - als deren Vertreter er die Serie GAME OF Thrones anführt (Z. 892) - kategorisiert er als eine Serie mit einer durchgeplanten „STOry line“ (Z. 893), die zu einer gleichbleibenden Spannung führe (Z. 898). Die zweite Serienart - prototypisch von ihm mit THE WALKING DEAD symbolisiert - kategorisiert er dagegen als eine, die ursprünglich nur für eine Staffel geplant und erst dann weiterentwickelt wurde, als sie Erfolg hatte (Z. 899-904). Gemeinsam mit Verena schreibt Ole in diesem Fall der Serie einen konstanten Qualitätsverlust zu (Z. 905-911). Ole bedient sich hier explikativen Kontrastes (Deppermann 2005; vgl. Abschnitt 3.2.3), indem er Produktionsentscheidungen im Hinblick auf den eigenen Rezeptionsanspruch miteinander vergleicht und begründet. Dabei führt er einen transseriellen Vergleich durch, der einzelne, konkrete Serien als exemplarische Repräsentationen für übergeordnete Typen modelliert. Daran wird nicht nur die evaluative Ausrichtung seiner Positionierung deutlich, 
sondern auch die epistemischen Implikationen: Ohne ausreichend fundiertes Wissen könnte er seine Position nicht begründen. Somit positioniert Ole sich als Experte, der sich einerseits durch seine $\mathrm{K}^{+}$-Position von den anderen Gruppenmitgliedern abgrenzt und sich andererseits über die geteilte Bewertung mit Verena vergemeinschaftet.

\subsubsection{Rekonstruieren und Inszenieren}

Die Beschreibung dieser Ressource orientiert sich zum einen an den in Abschnitt 3.2.1 herausgearbeiteten Formen der biographischen und narrativen Positionierung, d.h. sie bezieht sich auf von den Teilnehmenden berichtete Rezeptionserfahrungen oder -situationen (vgl. dafür v.a. Abschnitt 8.2.4.1 und 8.2.4.3). Zum anderen bezieht sie sich auch auf berichtende und performative Gesprächsbeiträge, mit denen die Teilnehmenden sich auf die Medienwelt bezogen oder das eigene Erleben bzw. Verhalten in Bezug auf die Medienwelt positionieren. Rekonstruktionen sind von allgemeinen Bezugnahmen auf Aspekte des Gegenstands insofern abzugrenzen, als sie eine strukturierende Selektion narrativer bzw. ästhetischer Elemente - oft in Relation zu der äußernden Person selbst - beinhalten. Sie können den Plot (Beispiel 19), den Handlungsverlauf einer einzelnen Episode oder einer Szene (Beispiel 27) und/oder das eigene Rezeptionserleben (Beispiel 43) betreffen.

Inszenieren bedeutet, dass die Teilnehmenden nicht nur darstellend oder berichtend über die Handlung oder eine Figur sprechen, sondern für eine kurze Zeit performativ mit der Stimme einer Figur sprechen oder eine Handlung mit dem Körper ausführen. Die Unterscheidung ist vergleichbar mit den von Hausendorf und Quasthoff (2005) beschriebenen Jobs des Elaborierens und Dramatisierens beim Erzählen. Allerdings handelt es sich beim Rekonstruieren dezidiert nicht nur um narrativ ausgestaltete, sondern auch berichtende Äußerungen. Inszenieren ist in den Daten seltener zu finden, was vermutlich auf die Rahmung der Situation als öffentlich (Abschnitt 6.1.2) zurückzuführen ist. Stattdessen greifen die Teilnehmenden eher auf die von Keppler (1989) ebenfalls im Rahmen von Tischgesprächen herausgestellten Kurzverweise oder Belehrungen zurück, um geteiltes Wissen zu aktualisieren oder epistemische Asymmetrie zu beheben. Genauso können Rekonstruktionen auch als Belege in Argumentationen herangezogen oder als „first“ oder „second stories“ (Sacks 1992: 765) entfaltet werden. Das folgende Fallbeispiel enthält sowohl Rekonstruieren als auch Inszenieren als Ressourcen:

(12) und hat gegen ne SCHEUne gekämpft.

$\begin{array}{lll}089 & \text { JOH: junge das ist die !DÜM!mste [sendung die } \\ 090 \text { VER: } & {[<<\text { sehr hoch>nei::n;>] es [gibt.] }} \\ 091 \text { SON: } & \text { [was ] pasSIERT }\end{array}$


da.

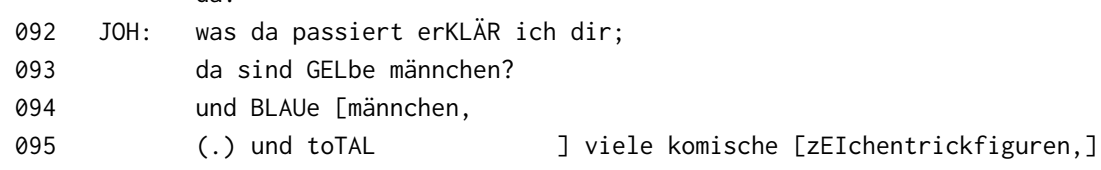

096 SEV: [das ist_n HUND; ]

097

Johanns evaluative Kategorisierung als ,!DÜM!mste sendung die es gibt.“(Z. 089) bezieht sich auf die Serie Adventure Time. Ausgelöst durch Sonjas etablierten Zugzwang zur Behebung einer epistemischen Asymmetrie: „was pasSIERT da; “ (Z. 091) beginnt Johann - nach einem metakommunikativen Kommentar, der die folgenden Äußerungen als belehrend rahmt - damit, Handlungselemente der Serie auf einer eher allgemeinen Ebene zu rekonstruieren (Z. 092-102). Trotz der auf den ersten Blick epistemisch ausgerichteten Positionierung zum Schließen einer 
Wissenslücke zwischen den Teilnehmenden liegt dennoch der Schwerpunkt auf Bewertungen. Denn Johann lädt primär zum gemeinsamen Vergnügen und Belustigung ein, wodurch er sich mit denjenigen, die ebenfalls zur Scherzinteraktion beitragen, vergemeinschaftet (Z. 103-105). Verenas frotzelnder Kommentar ,hast du das überhaupt geGUCKT, “ (Z. 097) - der durchaus seine epistemische Autorität zur Bewertung in Frage stellen könnte (vgl. dazu Analysen in Abschnitt 7.1.3) wird ignoriert.

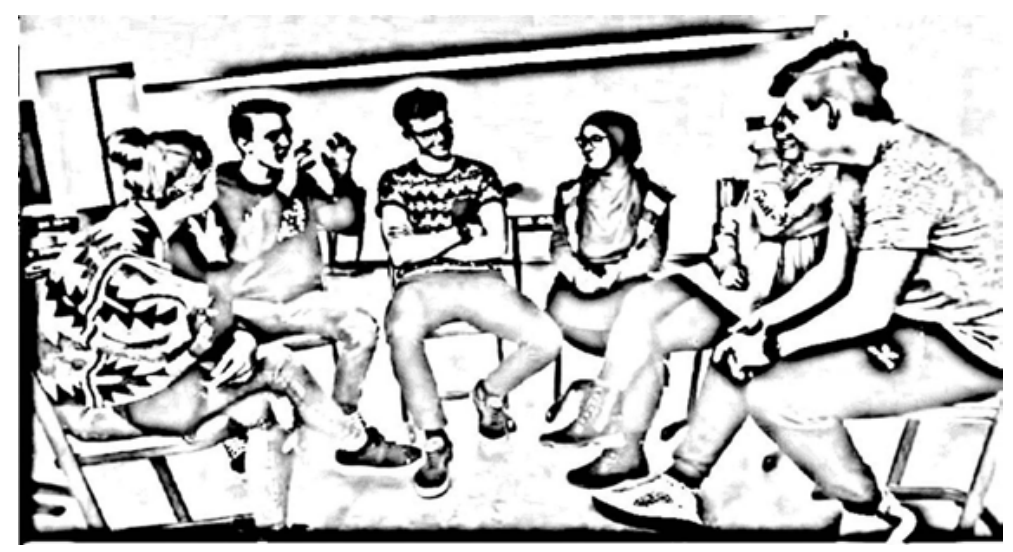

Abb. 6.3: JOH: und die scheune hatte ZWEI augen,

Stattdessen fährt Johann mit seiner Rekonstruktion fort und begibt sich auf die Ebene der Handlungselemente einer spezifischen Episode, die er rekonstruiert (Z. 106-121). An dieser Stelle greift er zu inszenierenden Elementen. Wie in Abbildung 6.3 visualisiert, formt Johann mit seinen Händen die Augen der Scheune, während er formuliert: „und die scheune hatte ZWEI augen, “ (Z. 120), so dass er kurzzeitig zur Veranschaulichung die Rolle der Figur der Scheune einnimmt. Auch damit evoziert er einerseits Belustigung und frotzelnde Kommentare (Z. 122-123), stützt aber zugleich epistemisch seine evaluative Argumentation, da er zuvor die Serie als „größte[n] QUATSCH;“ (Z. 079) bezeichnet hatte. Über die Scherzinteraktion wird hier auch Vergemeinschaftung hergestellt, an der Verena sich zwar teilweise beteiligt, sich dann durch ihre Kommentare aber auch davon distanziert. Über die hier beschriebenen Ressourcen wird analytisch einbezogen, wie die Teilnehmenden ihre epistemische und/oder evaluative Haltung zu dem Gegenstand signalisieren. Je nachdem, wie die Ressourcen interaktiv aufgegriffen und behandelt werden, können sie Positionierungsdynamiken und damit das Herstellen von Vergemeinschaftung und Distinktion ermöglichen. 


\subsubsection{Der Gegenstand als sequenzstrukturierendes Mittel}

In diesem Abschnitt wird dargestellt, welche Funktion dem Gegenstand für die Gesprächsorganisation zukommt. Während in Abschnitt 6.1 gezeigt wurde, dass die Teilnehmenden die Situation als aufgabenorientierte Interaktion behandeln, wird daran anknüpfend im Folgenden aufgezeigt, dass sie die Gespräche entlang der thematischen Vorgabe der Instruktion strukturieren und organisieren. Die meisten Gesprächssequenzen werden dadurch initiiert, dass sich der Gesprächsgegenstand ändert, zum Beispiel indem über eine andere Serie, Rezeptionsvorlieben o.Ä. gesprochen wird. Die Daten wurden dafür einer detaillierten sequenziellen Analyse im Sinne des next-turn-proof-procedures (Abschnitt 4.1) unterzogen, um die Sequenzstrukturierung identifizieren zu können (vgl. auch Abschnitt 5.2).

Darüber wurde herausgearbeitet, dass die Interagierenden eine neue Sequenz häufig dadurch initiieren, dass sie einen neuen thematischen Aspekt des Gesprächsgegenstands als Positionierungsobjekt (Abschnitt 6.5.1) in die Interaktion einbringen. In Anlehnung an die rekonstruierten kommunikativen Aufgaben (Abschnitt 6.3) indizieren die Teilnehmenden den Gegenstand, indem sie ihm gegenüber eine epistemische und/oder evaluative Haltung kommunizieren. Dadurch, dass die anderen Beteiligten im Folgenden ebenfalls epistemische und/oder bewertende Äußerungen anschließen, entwickeln sich Positionierungssequenzen. Somit wird in dieser Arbeit in Anlehnung an Wiesner (2014) von Gegenstandspositionierungen gesprochen (vgl. Merkmal (4) in den Abschnitten 3.2.5 und 3.3). Damit wird auch der potentiellen Ubiquität von Positionierungen (Aspekt (5); vgl. Abschnitt 3.3) analytisch begegnet, indem sie über die epistemische und/oder evaluative Indizierung eines (neuen) Gegenstands oder Gegenstandsaspekts identifiziert werden.

Diese Indizierung wird für diese Studie als Etablieren eines Positionierungsobjekts bezeichnet. Damit kann eine explizite oder implizite Selbst- und/oder Fremdpositionierung und/oder ein Positionierungsangebot einhergehen, je nachdem, wie eindeutig die Haltung auf der interaktiven Oberfläche erkennbar ist. Zudem können die Positionierungen unterschiedliche Grade an Intensität aufweisen (Aspekt (6); vgl. Abschnitt 3.3 und 6.4). Die sich in den meisten Fällen anschließende Aushandlungssequenz beinhaltet dann Folgepositionierungen, die gleichermaßen epistemisch und/oder evaluativ auf das Objekt bezogen sein können und sich üblicherweise an der initialen Positionierung ausrichten, also Alignment im Sinne des stance-Dreiecks (vgl. Abschnitt 3.2.4) signalisieren. Folgepositionierungen finden zwar überwiegend statt, es gibt aber auch Fälle, in denen erste Positionierungen nicht aufgegriffen werden. Nach Beendigung einer Sequenz kann ein neues serienbezogenes Positionierungsobjekt etabliert werden oder die Teilnehmenden machen etwas anderes zum Thema, wie z.B. die Aufnahmesitua- 
tion, Vorkommnisse in der Schule o.Ä. So lässt sich folgende Sequenzstruktur empirisch rekonstruieren:

- Etablieren eines Positionierungsobjekts

- Erste Positionierungen

- Anzeigen der eigenen evaluativen und/oder epistemischen Haltung8 $\rightarrow$ explizite Selbstpositionierung

- Zuschreiben einer epistemischen (seltener: evaluativen) Haltung an (mindestens) eine andere Person

$\rightarrow$ explizite Fremdpositionierung, ggf. auch implizite Selbstpositionierung

- Erfragen der epistemischen und/oder evaluativen Haltung von (mindestens) einer anderen Person

$\rightarrow$ implizite Selbstpositionierung

- Folgepositionierungen

- Etablieren weiterer Selbst- und/oder Fremdpositionierungen

- Aufrechterhalten von Selbst- und/oder Fremdpositionierungen

- Modifizieren von Selbst- und/oder Fremdpositionierungen

- Abschließen und ggf. Überleiten zu einem neuen oder modifizierten Positionierungsobjekt

Im Folgenden werden die einzelnen Aspekte detaillierter und anhand von exemplarischen Fallbeispielen beschrieben. Mit dem Etablieren eines Positionierungsobjekts geht häufig schon eine erste Positionierung der sprechenden Person durch Anzeigen der eigenen Haltung einher. Das ist etwa in Beispiel 17 der Fall, wenn Ali eine neue Sequenz an die vorherige Sequenz kontextualisierend anschließt: ,so SOwas ${ }^{9}$ würd mich AUCH ziemlich Anprechen, aber was mich zum beispiel am MEISten anspricht, sind filme so die im MITtelalter spielen oder sowas; sowas wie game of THRONES die haben (.) so $n$ mittelal, mit MITtelalterliche (.) äh:m; (-) halt, (.) mit MITtelalterlich dargestellt; =und das MAG ich; (MNG13, Z. 232-239). Er zeigt seine positive evaluative Haltung in Bezug auf mittelalterliche Serien an und etabliert damit zugleich GAME OF THRONES als Vertreter dieses Genres als Positionierungsobjekt, woraufhin sich Folgepositionierungen der Gruppenmitglieder anschließen (Z. 240-258).

Eine erste Positionierung kann auch mit dem Zuschreiben einer Haltung an die Gruppenmitglieder beginnen. So rahmt beispielsweise Burim, gefragt von Lara, was er „NOCH,“ (EMS51, Z. 064) gucken würde, seine Präferenzen folgenderma-

8 Hier synonym zu stance gebraucht; vgl. Ausführungen in Abschnitt 3.2.4.

9 Anmerkung: „sowas“ bezieht sich auf den vorher von anderen Gruppenmitgliedern erläuterten Plot der Serie ICERDE. 
ßen: „äh:m, (.) also meine LIEBlingsserie, (.) aber die WERdet ihr-die werdet ihr zu !TAU!sendprozent nicht kennen, (.) ist OZ;=HÖLle hinter gittern; “ (Z. 065-070) ${ }^{10}$. Er schreibt also den anderen Teilnehmenden mit starker Gewissheit einen niedrigen epistemischen Status in Bezug auf die Serie Oz - HöLLE HINTER GITTERN als Positionierungsobjekt zu, was die Gruppenmitglieder interaktiv bestätigen, indem sie Burim zur Elaborierung und Verständnissicherung auffordern (Z. 0072-076). Zugleich wird seine eigene positive evaluative und epistemisch hohe Haltung durch die Verwendung des Lexems „LIEBlingsserie“ (Z. 065) deutlich.

Mit dem Erfragen einer Haltung kann eine erste explizite Selbstpositionierung vermieden werden. Damit sind Äußerungen wie Johanns Frage: „THUN, THUNdermans, (.) kennt ihr DIE zufällig?" (Anhang IX: OST11, Z. 220-221) gemeint. Damit kann eine Person es anderen Beteiligten bzw. der Gruppe überlassen, als erstes eine Haltung zu kommunizieren, d.h. sie macht ein Positionierungsangebot, das die nächsten Sprecher*innen in Form von Folgepositionierungen annehmen können. Einen besonderen Fall des Erfragens findet sich in Beispiel 8. Bilel etabliert die Serie SCRUBS zunächst indirekt als Positionierungsobjekt, indem er formuliert: „aber, (.) in letzter zeit guck ich auch eine ALte serie die gar nich mehr läUft; “ (Z. 176-177) und den Titel erst auf Nachfrage nennt (Z. 178-179). Auch seine Haltung zur Serie bleibt zunächst diffus - es kann zwar interferiert werden, dass er die Serie kennt und mag; das wird allerdings zunächst nicht explizit deutlich. So vermeidet er eine explizite Selbstpositionierung und überlässt es der Gruppe, als erstes eine Haltung zu kommunizieren, ohne sie dezidiert zu erfragen. Die Folgepositionierungen bedienen dieses Angebot und die Teilnehmenden etablieren diverse evaluative Selbstpositionierungen (Z. 180-192). Erst in dieser Sequenz beteiligt sich Bilel selbst und markiert seine eigene evaluative Haltung ebenfalls als positiv (Z. 185). In den meisten Fällen fällt mit dem Erfragen das Rederecht nach einer oder zwei Folgepositionierungen automatisch an die oder den erste ${ }^{\star} n$ Sprecher ${ }^{\star}$ in zurück. Das zeigt sich etwa an folgender Sequenz: Leni fragt die anderen Gruppenmitglieder, wie viele Staffeln sie an einem Wochenende schauen (Anhang IV: MNG11, Z. 879-882). Als wenig später die anderen Gruppenmitglieder zwar geantwortet haben, Leni selbst aber noch keine Prognose abgegeben hat, gibt sie dafür einen account (vgl. zur Begriffsklärung Abschnitt 4.1): „=ich sag euch gleich wie viel ICH dazu11 gebraucht hab;“ (Z. 903; vgl. auch die Einführung in Abschnitt 8.2.4.3).

Die präzise Analyse der ersten Positionierung ist insofern relevant, als die Folgepositionierungen sich dazu alignieren. Sie zeigen an, ob und inwiefern die

10 Vgl. zur Analyse der Funktion seiner hier vorgenommenen Positionierungen im Interaktionsverlauf Abschnitt 8.1.4 und Beispiel 38.

11 Damit bezieht sie sich auf die Serie One TREe Hill. 
anderen Gruppenmitglieder die Serie oder einen serienbezogenen Aspekt in Relation zu der ersten Positionierung kennen und bewerten. Darüber können sie dann Zustimmung oder Widerspruch ausdrücken, was die Grundlage für die Herstellung von Vergemeinschaftung bzw. Distinktion bildet. Es gibt Fälle, in denen eine erste Positionierung zu einem neuen Positionierungsobjekt interaktiv nicht oder kaum weiter aufgegriffen wird, wie beispielsweise Sevcans Bewertung: „ich mag bob der BAUmeister; “ (Anhang IX: OST11, Z. 128)12. Teilweise können Positionierungen im Laufe des Interaktionsverlaufs immer wieder aktualisiert werden; so wird beispielsweise Tims Positionierung gegen GAME of THRONEs direkt zu Beginn der Diskussion (vgl. Beispiel 6 und die Analysen in Abschnitt 9.1.1) häufig aufgegriffen und als relevant markiert.

Auch wenn das Sequenzmuster empirisch rekonstruiert wurde, kann es nicht immer eine eindeutige und trennscharfe Einordnung von Fällen leisten. Es fungiert als Schablone für die Analyse von Positionierungspraktiken und -orientierungen in den Kapiteln 7 und 8, um damit sowohl die Dynamik als auch die Interaktivität und Relationalität von Positionierungen (vgl. Aspekte (2) und (3) in Abschnitt 3.3) analytisch erfassen und beschreiben zu können.

\subsection{Zusammenfassung: Analytischer Zugriff auf Positionierungen im Kontext von Serienkommunikation}

Im Folgenden wird der analytische Zugriff auf die Daten erläutert. Die Analyse orientiert sich an dem stance-Dreieck von Du Bois (2007) (vgl. Abschnitt 3.2.4), wird allerdings für die vorliegende Studie modifiziert und erweitert. Die in Abschnitt 3.3 herausgearbeiteten Merkmale von Positionierungen - (1) Kontextgebundenheit, (2) Interaktivität und Relationalität, (3) Dynamik, (4) Gegenstandsbezogenheit, (5) (potentielle) Ubiquität, (6) Explizitheit und Intensität, (7) Räumlichkeit - werden mit der empirischen Rekonstruktion von Positionierungen, wie sie in Kapitel 6 erläutert wurden, kombiniert. Die Gebundenheit an den Kontext (Merkmal (1)) wird als interaktiv hervorgebracht verstanden, wobei die in Abschnitt 6.1.2 beschriebenen Aspekte - Öffentlichkeit, Institutionalität, Kooperativität - Berücksichtigung finden.

Um der gegebenen Mehrparteieninteraktion gerecht zu werden, wird die von Du Bois angenommene Dyade (bei Du Bois: Subject 1 und Subject 2) dahingehend erweitert, dass der zweite Interaktionspart auch mehrere Personen umfassen

12 Die hier saliente Praktik des (nicht) erfolgreichen Etablierens eines Positionierungsobjekts wird in Abschnitt 7.2.3 analysiert. 
kann. Zudem können sich auch aus mehreren Teilnehmenden bestehende, sich selbst und einander positionierende Allianzen entwickeln. Die so für das Merkmal der (2) Interaktivität und Relationalität relevante Beziehung zwischen mehreren Interagierenden und einem Kommunikationsgegenstand wird folgendermaßen modelliert: Die Interaktionsparteien positionieren sich und/oder andere, indem sie ihre evaluative Haltung - darin folgt die Darstellung Du Bois' Überlegungen und/oder ihre epistemische Haltung dazu anzeigen (vgl. die empirisch rekonstruierten kommunikativen Aufgaben in Abschnitt 6.3), die wiederum im Hinblick auf Explizitheit und Intensität (Merkmal (6)) analysiert werden. Als Ressourcen für Positionierungen können die in Abschnitt 6.5.2 rekonstruierten Varianten Kategorisieren und Zuschreiben, Kontrastieren und Vergleichen, Rekonstruieren und Inszenieren - fungieren.

Diese Ressourcen sind typisch für das Sprechen über den per Instruktion (Abschnitt 6.1.1) vorgegebenen Gesprächsgegenstand, der für diese Studie als Positionierungsobjekt modelliert wird. In Abschnitt 6.5.3 wurde gezeigt, dass dem Positionierungsobjekt die Funktion zukommt, die Gespräche sequenziell zu strukturieren (Merkmal (4): Gegenstandsbezogenheit und Merkmal (5): Ubiquität). Dabei kann es dynamisch variieren von konkreten Serienprodukten bis zu abstrakteren serienund rezeptionsbezogenen Aspekten. Davon hängt ab, ob sich die Beteiligten mit lokaler bzw. globaler Reichweite positionieren (vgl. Abschnitt 6.5.1). Es wird auch berücksichtigt, dass sich die Positionierungen nicht nur aufeinander beziehen (durch das Alignment zwischen den Interagierenden), sondern auch im Laufe der Gesprächsdynamik verändern und zwischen den Interagierenden ausgehandelt werden können (Merkmal (3): Dynamik). Dadurch stellen die Interaktionsbeteiligten Gemeinschaft bzw. Distinktion (Abschnitt 6.2.2) her - unter der Voraussetzung der Partizipation am Gespräch (Abschnitt 6.2.1).

Um Prozesse der Vergemeinschaftung und Distinktion auch visuell zu erfassen, wird auf Merkmal (7) - Räumlichkeit - in metaphorischer Art und Weise zurückgegriffen: Die Teilnehmenden positionieren sich epistemisch und evaluativ in einem ,Feld', das in Form eines zweidimensionalen Koordinatensystems dargestellt wird (vgl. Abschnitt 6.4). Dieses Schema erlaubt es, den Zusammenhang zwischen epistemischen und evaluativen Positionierungen der Interagierenden - in Bezug auf eine konkrete Serie, d.h. mit lokaler Reichweite - innerhalb einer Sequenz visuell abzubilden. Auf der X-Achse kann so der rekonstruierte epistemische Status von Personen (von $\mathrm{K}^{+}$bis $\mathrm{K}^{-}$) und auf der Y-Achse deren Bewertung (positiv/negativ) eingeordnet werden (Abbildung 6.1), so dass ein Überblick entsteht, welche Interagierenden sich eher als (un-)wissende Unterstützer^innen bzw. Kritiker`innen einordnen lassen. Mit globaler Reichweite werden dagegen Bezugnahmen auf übergeordnete Aspekte erfasst, die über dieses Koordinatensystem nicht abgebildet 
werden können. Wie beides zusammenhängend bedacht werden kann, wird in Kapitel 9 erläutert.

Durch die Unterscheidung von lokalen und globalen Positionierungen werden unterschiedliche Richtungen der Analyse verfolgt. Für den lokalen Aspekt ist von Bedeutung, dass epistemische und evaluative Haltungen nicht als getrennte Formen der Positionierung betrachtet, sondern in ihrem Zusammenspiel untersucht werden (Kapitel 7). So kann darüber analysiert werden, welche Positionierungspraktiken Teilnehmende nutzen, um epistemische Autorität zur Bewertung zu beanspruchen, wenn sie eine Serie gut kennen, sich also als $K^{+}$positionieren (wie Josefine und Emilia in Beispiel 9; vgl. dazu Abschnitt 7.1). Daneben lohnt es sich, auch diejenigen Teilnehmenden in den Blick zu nehmen, die sich als $K^{-}$positionieren, aber zugleich Gesprächspartizipation anstreben (wie Martha in den Beispielen 8 und 9; vgl. dazu Abschnitt 7.2). Weiterhin soll empirisch ausdifferenziert werden, welche Rolle der Gesprächsgegenstand für Positionierungen spielt. Da je nach Abstraktionsgrad des Positionierungsobjekts sich die Ausrichtung der Positionierung (lokal vs. global) unterscheidet, wird in einem zweiten Analyseschritt auf der Grundlage der Positionierungspraktiken herausgearbeitet, welche Aspekte Teilnehmende in Bezug auf Serien grundsätzlich in welcher Form thematisieren (Kapitel 8). Dazu wird rekonstruiert, an welchen Wissensbeständen und Werturteilen sie sich orientieren, wenn sie über eher medienbezogene (Abschnitt 8.1) und über rezeptionsbezogene (Abschnitt 8.2) Aspekte diskutieren und sich diesbezüglich positionieren. Damit greift die Arbeit die Überlegung von Keppler auf, Medien fungierten als „,Relais‘ zwischen privaten und öffentlichen Orientierungen“ (Keppler 1994: 263-264) (vgl. Abschnitt 2.2.3). 